\title{
Anisotropic Cathodic Corrosion of Gold Electrodes in the Absence and Presence of Carbon Monoxide
}

\author{
Stefan J. Raaijman, ${ }^{\dagger}$ Nakkiran Arulmozhi, ${ }^{\dagger}$ and Marc T. M. Koper* \\ Cite This: J. Phys. Chem. C 2020, 124, 28539-28554 \\ Read Online
}

ABSTRACT: In this work, we investigate gold surface dissolution under cathodic potentials as a function of crystal orientation using cyclic voltammetry, scanning electron microscopy, and atomic force microscopy to determine to what extent cathodic corrosion is anisotropic in nature. Additional experiments were performed to gain insights into the effect of the presence of $\mathrm{CO}$ on cathodic corrosion. Carbon monoxide experiments were conducted to investigate the effect of more realistic reaction conditions on cathodic corrosion as they might be found in gold-driven $\mathrm{CO}_{2}$ electrolyzers, although the effects of the presence of $\mathrm{CO}_{2}$ (reactant) and carbonate salts (typically present in the reaction medium) were

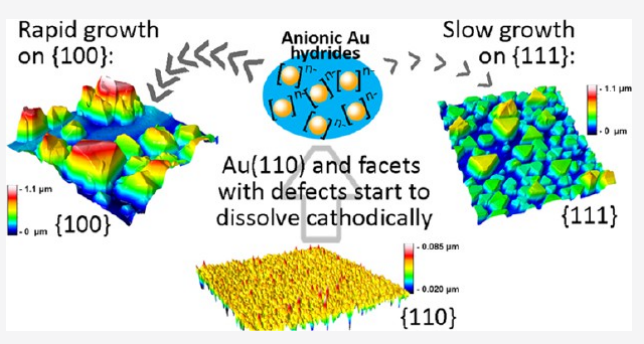
excluded from this study. It was found that cathodic corrosion of gold is strongly anisotropic, with stepped surfaces and the $\{110\}$ plane being most susceptible to dissolution, whereas the $\{111\}$ and $\{100\}$ planes were much more resilient to dissolution. On these more stable surfaces, nanocrystallite growth was observed instead, which we hypothesize originates from redeposition of the gold that dissolves from the more corrosive, more open, and/or defective facets. Finally, the presence of carbon monoxide was found to slightly enhance the rate of surface change, as evidenced by increases in charge in cyclic voltammograms after corrosion and an earlier onset of crystallite growth on the $\{111\}$ and $\{100\}$ planes, which we ascribe to enhanced gold mobility due to the strong bond formed between $\mathrm{CO}$ and gold in alkaline media.

\section{INTRODUCTION}

With the predicted strong growth of renewable energy sources, ${ }^{1}$ their intermittency will become increasingly problematic. One option for dealing with the intermittency is to electrochemically generate value-added products at times when the supply supersedes demand. The electrochemical conversion of carbon dioxide and water or the $\mathrm{CO}_{2}$ reduction reaction $\left(\mathrm{CO}_{2} \mathrm{RR}\right)$ would make a suitable contender for this role as it can yield a plethora of valuable products in the form of hydrocarbons, carbohydrates, and (inorganic) acids. However, the $\mathrm{CO}_{2} \mathrm{RR}$ requires a suitable catalyst to facilitate the efficient conversion of electrical work into chemical products. Fortunately, catalysts exist that are capable of reducing carbon dioxide to economically interesting products with good selectivity and efficiency: gold $^{2}$ and silver $^{3}$ are capable of forming carbon monoxide with high selectivity, indium $^{4}$ and palladium ${ }^{5}$ are selective for the formation of formic acid, and depending on the exact conditions, copper can produce ethylene ${ }^{6}$ or ethanol $^{7}$ with very reasonable efficiencies.

However, the applicability of a catalyst is subject to stringent performance tolerances for it to be economically viable. Two important metrics are catalyst cost and lifetime ${ }^{8}$ because replacement of the spent catalyst requires capital and leads to process downtime, reducing overall profitability. Under $\mathrm{CO}_{2} \mathrm{RR}$ relevant conditions (negative potentials, high current flows, high local $\mathrm{pH}$ values, and large amounts of the product), metals can undergo substantial morphological restructuring and possibly dissolve because of cathodic corrosion. ${ }^{9}$ Cathodic corrosion is a degradation pathway that might prove quite relevant for catalyst stability and long-term performance in $\mathrm{CO}_{2}$ electrolyzers. This phenomenon has been mainly studied on platinum electrodes, ${ }^{9-12}$ leading to the formation of nanoparticles in solution and etch pits on the metal surface, resulting in the generation of specific surface sites. ${ }^{12}$ It has been hypothesized that these pits originate from atom clusters being expelled from the surface via the formation of a negatively charged ternary metal hydride complex. ${ }^{11}$ The process itself has been shown to depend strongly on cation identity and concentration, and there appears to be good agreement between the onset of corrosion and the specific potential window where adsorption of a full monolayer of hydrogen becomes energetically favorable. ${ }^{11}$

At present, $\mathrm{CO}_{2}$ electrolyzers focusing on $\mathrm{HCOOH}$ or $\mathrm{CO}$ production are regarded as closest to commercialization, with CO having a larger market. ${ }^{13}$ The best catalysts for producing $\mathrm{CO}$ are silver and gold, both supporting high selectivity at low overpotentials. ${ }^{2,3,14}$ However, both metals have been reported to undergo cathodic corrosion. ${ }^{\text {? }}$

Received: September 21, 2020

Revised: December 2, 2020

Published: December 17, 2020

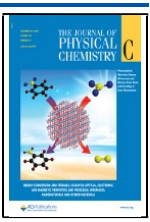


Therefore, we explore in this paper the cathodic corrosion of gold single-crystal electrodes, showing evidence for a strong anisotropic effect with regard to both pitting and nanocrystal formation. The choice to study gold is based on the fact that the field of gold single-crystal electrochemistry is mature and expansive ${ }^{15-17}$ and experimentally more accessible. ${ }^{18,19}$ There already exist literature studies on its cathodic corrosion behavior (for polycrystalline surfaces), $, 111,20$ showing that gold exhibits a tendency to form (nano)crystallites on the surface. ${ }^{20}$ An additional motivation for the work in this paper is that cathodic corrosion has been studied predominantly under HER conditions, as opposed to $\mathrm{CO}_{2} \mathrm{RR}$ conditions. ${ }^{21}$ Trends in macroscopic pitting and deposition phenomena as a function of step type and step density were investigated on spherical single crystals $[\mathrm{Au}($ sphere $)]$, while cut crystals $[\mathrm{Au}(\mathrm{hkl})]$ enabled us to deconvolute electrochemical signals from $\mathrm{Au}$ (sphere) crystals. Finally, experiments were conducted with the addition of gaseous $\mathrm{CO}$ to the electrochemical cell as a means to approximate realistic $\mathrm{CO}_{2} \mathrm{RR}$ conditions. ${ }^{6}$

\section{METHODS}

2.1. Electrodes, Electrolytes, and Electrochemical Cells. The experimental work was conducted using monocrystalline bead-type $\mathrm{Au}(111), \mathrm{Au}(110)$, and $\mathrm{Au}(100)$ electrodes (icryst, oriented to $\left.\leq 0.1^{\circ}\right)$, and $\mathrm{Au}$ (spherical) working electrodes (WEs) prepared using the flame fusion method. $^{22-24}$ Prior to each measurement, spherical single crystals were entirely regrown and flame-annealed immediately prior to measurement, while cut crystals were annealed at ca. $950{ }^{\circ} \mathrm{C}$ in a pure hydrogen flame for at least $5 \mathrm{~min}$. A gold mesh (99.999\%) was used as a counter electrode (CE), and a reversible hydrogen electrode (RHE) (Hydroflex, Gaskatel) was used as a reference electrode (RE). Two types of custommade electrochemical cells were employed in this study, namely, a Pyrex glass cell and a fluorinated ethylene propylene (FEP) cell. The two-compartment Pyrex glass cell was used in all cyclic voltammetry $(\mathrm{CV})$ measurements in acid media, and the FEP cell was employed in cathodic corrosion measurements in alkaline media. The FEP cell consists of a single compartment with ca. $3 \mathrm{~cm}$ of distance between the $\mathrm{CE}$ and the WE surface. The $\mathrm{H}_{2} \mathrm{SO}_{4}$ (96\%, Ultrapur, Millipore) and $\mathrm{NaOH}$ (30\%, Suprapur, Millipore) electrolyte solutions were prepared using ultra-high-purity (UHP) Milli-Q water (Millipore; resistivity $\geq 18.2 \mathrm{M} \Omega \mathrm{cm}$ ). Glassware and plasticware were cleaned by storing them in aqueous permanganate solution $\left[0.5 \mathrm{M} \mathrm{H}_{2} \mathrm{SO}_{4}\right.$ (96\%, ACS reagent, Honeywell) and $1 \mathrm{~g} / \mathrm{L} \mathrm{KMnO}_{4}$ (99\%, ACS reagent, Sigma Aldrich)]. Prior to the experiment, the residue was removed by rinsing with Milli-Q water and washing with diluted piranha solution (3:1 v/v mix of $\mathrm{H}_{2} \mathrm{SO}_{4}(96 \%) / \mathrm{H}_{2} \mathrm{O}_{2}(30 \%)$, diluted with water) to remove manganese dioxide and the permanganate residue. Next, glassware was boiled five times in Milli-Q water. Ultra-high-purity argon (Linde, 6.0) was passed through and above the electrolyte to remove any dissolved gases. Prior to electrode-electrolyte contact, argon flow through the electrolyte is stopped to avoid noise, while the argon flow above the electrolyte is maintained. After annealing the monocrystalline gold electrodes, the hot electrode is subsequently transferred to the electrochemical cell and cooled in an argon atmosphere. Once the electrode was cooled to room temperature, the so-called hanging meniscus electrode-electrolyte connection was employed for establishing electrochemical contact of the $\mathrm{Au}(111), \mathrm{Au}(110)$, and $\mathrm{Au}(100)$ electrodes. In the case of $\mathrm{Au}($ spherical $){ }^{25}$ the electrode was carefully placed in the electrolyte so that the monocrystalline gold sphere was entirely submerged. All electrochemical experiments were conducted using a BioLogic SP-300 potentiostat using proprietary software. All surfaces were normalized assuming $390 \mu \mathrm{C} \mathrm{cm}^{-2}$ (the value assigned to polycrystalline gold), which was deemed to best represent the imperfect nature of the cut single crystals, and the fact that many different sites are present on spherical single crystals. ${ }^{26}$ Cyclic voltammograms obtained post corrosion were normalized using the surface area calculated for the blank obtained prior to corrosion.

2.2. Cathodic Corrosion Studies. The cathodic corrosion studies were performed at $\mathrm{Au}(111), \mathrm{Au}(110), \mathrm{Au}(100)$, and $\mathrm{Au}($ spherical) electrodes via application of a constant potential $\left(E_{\mathrm{p}}\right.$ in the range between $-1.10 \mathrm{~V}$ and $\left.-1.30 \mathrm{~V}_{\mathrm{RHE}}\right)$ for a controlled polarization time $\left(t_{\mathrm{p}}\right.$, with $\left.2.5<t_{\mathrm{p}}<7.5 \mathrm{~min}\right)$ in 10 $\mathrm{M} \mathrm{NaOH}$ at room temperature. These conditions were chosen on account of the fact that the least cathodic potential and the shortest time were close to the observable onset for surface restructuring from scanning electron microscopy (SEM), whereas the upper limit (the most cathodic potential and the longest time) showed large surface changes but could still be related to the initial faceting. Specifically, shorter times at less cathodic potentials were not tried as the current least corrosive conditions $\left(2.5 \mathrm{~min}\right.$ at $\left.-1.1 \mathrm{~V}_{\mathrm{RHE}}\right)$ exhibited only minor signs of change in SEM images. More strongly cathodic potentials (5 min at $-1.5 \mathrm{~V}_{\mathrm{RHE}}$, not shown) were initially tested but resulted in such drastic changes that it became impossible from SEM to correlate the initial substrate structure to the morphology observed after corrosion. Some tests were conducted for longer times ( $1.5 \mathrm{~h}$ at $-1 \mathrm{~V}_{\mathrm{RHE}}$, figure $\left.\mathrm{S} 4\right)$ as well, but corrosion for such long times resulted in significant issues with surface cleanliness, being detrimental to experiment reproducibility. The electrolyte was used for either a maximum of $10 \mathrm{~min}$ of corrosion time or a total of three separate measurements (depending on which threshold was reached first), after which it was discarded and the cell was thoroughly rinsed, and the fresh electrolyte was added. All corrosion measurements were conducted using a freshly prepared electrode surface. For all corrosion experiments, argon or $\mathrm{CO}$ was bubbled through the solution before and during the experiment. Cathodic polarization studies were conducted with the cell submerged in a water bath at room temperature with a magnetic stirrer (in the water bath) to prevent electrolyte heating.

Figure 1 displays a potential versus time profile that illustrates the sequence of steps (a through e) involved in the corrosion studies.

- First (step a), a cyclic voltammogram is measured in the $0.05 \mathrm{M} \mathrm{H}_{2} \mathrm{SO}_{4}$ electrolyte in the potential window of 0 $\leq E \leq 1.65 \mathrm{~V}_{\mathrm{RHE}}$ at a scan rate of $\nu=80 \mathrm{mV} \mathrm{s}^{-1}$ to characterize the initial surface.

- Second (step b), the electrode is rinsed with Milli-Q water and flame-annealed in either a hydrogen flame of ca. $950{ }^{\circ} \mathrm{C}$ for $5 \mathrm{~min}$ (cut crystals) or a butane flame until the gold surface is faintly red hot for $5 \mathrm{~min}$ (spherical crystals) to remove the defects generated in step a.

- Third (step c), the electrode is immersed in $10 \mathrm{M}$ $\mathrm{NaOH}$ (at a controlled potential of $0 \mathrm{~V}_{\mathrm{RHE}}$ ) but not in a hanging meniscus configuration in this instance. The application of a constant polarization potential from $E=$ 


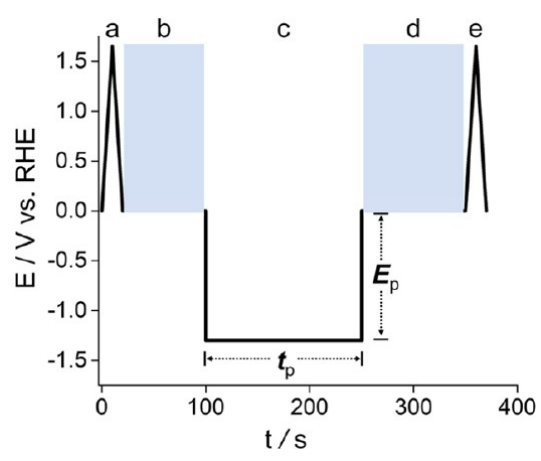

Figure 1. Potential program employed in the cathodic corrosion studies. Step a: CV in the sulfate adsorption/desorption region and the gold oxidation/reduction region in $0.05 \mathrm{M} \mathrm{H}_{2} \mathrm{SO}_{4}$ at a scan rate of $\nu=80 \mathrm{mV} \mathrm{s}^{-1}$. Step b: cleaning the electrode in Milli-Q water and annealing for $>5 \mathrm{~min}$. Step c: cathodic corrosion in $10 \mathrm{M} \mathrm{NaOH}$ aqueous solution at a given potential $E_{\mathrm{p}}$ for a given time $t_{\mathrm{p}}$. Step d: cleaning the electrode with Milli- $Q$ water. Step e: CV in the sulfate adsorption/desorption region and the gold oxidation/reduction region in $0.05 \mathrm{M} \mathrm{H}_{2} \mathrm{SO}_{4}$ at a scan rate of $\nu=80 \mathrm{mV} \mathrm{s}^{-1}$.

$0.00 \mathrm{~V}_{\mathrm{RHE}}$ to a defined (85\%) iR compensated polarization potential limit $E_{\mathrm{p}}$ (in Figure $1, E_{\mathrm{p}}=-1.30$ $\mathrm{V}_{\mathrm{RHE}}$ ) for a defined polarization time $t_{\mathrm{p}}$ (in Figure $1, t_{\mathrm{p}}=$ $2.5 \mathrm{~min})$

- Thereafter (step d), the cathodically corroded crystal is rinsed with Milli-Q water for sodium hydroxide removal.

- Finally (step e), the electrode surface after corrosion is characterized again via $\mathrm{CV}$ as described in step a.

Because of the variation in the potential sensitivity of different facets with regard to adsorption of ions, $\mathrm{CV}$ in $\mathrm{H}_{2} \mathrm{SO}_{4}$ (in the window of $\left.0 \leq E \leq 1.65 \mathrm{~V}_{\mathrm{RHE}}\right)^{15-17}$ can be used to characterize changes in surface structure via comparison of $\mathrm{CV}$ profiles obtained prior to and after cathodic treatment.

2.3. Microscopic Analysis of Cathodic Corrosion. Surface roughening of the monocrystalline $\mathrm{Au}$ (spherical) electrodes brought about by cathodic corrosion was analyzed using SEM (Apreo SEM, Thermo Scientific) and atomic force microscopy (AFM, JPK Nanowizard 4). After the corrosion experiment, the monocrystalline Au electrode was carefully removed from the electrochemical cell and placed in a custom- made SEM sample holder. Then, the sample was rinsed under a stream of Milli-Q water to remove traces of the acid from the electrode. Subsequently, the monocrystalline $\mathrm{Au}$ (spherical) electrode was dried in a stream of $\operatorname{Ar}(\mathrm{g})$ and placed in the SEM/AFM chamber for analysis. SEM was performed under high-vacuum conditions $\left(<5 \times 10^{-6} \mathrm{mbar}\right)$, and the images were obtained at a beam setting of $10 \mathrm{kV}$ and $0.40 \mathrm{nA}$ using an Everhart-Thornley detector. The AFM measurements were conducted in the contact mode using a gold-coated tip (NANOSENSORS PPP-FMAuD; tip height: $10-15 \mu \mathrm{m}$, tip resonance frequency: $75 \mathrm{kHz}$, tip force constant: $2.8 \mathrm{~N} / \mathrm{m}$ ) at a scan rate of $0.4 \mathrm{~Hz}$. Crystals not being actively measured/used were affixed in a custom holder and stored in a plastic vial so as to minimize ambient exposure.

In order to identify the main surface facets in the $\mathrm{Au}$ (spherical) electrode, we compare the face-centered cubic (fcc) unit cell to an SEM image of the $\mathrm{Au}$ (spherical) electrode. Figure 2a shows an SEM image of an as-imaged monocrystalline $\mathrm{Au}$ (spherical) electrode, and Figure $2 \mathrm{~b}$ shows the fcc lattice structure of a Au single crystal, with the low Miller index facets depicted using different colors (i.e., $\{111\}$ in red, $\{100\}$ in blue, and $\{110\}$ in green). In the SEM image, stereographic triangles are superimposed using gray, dashed lines. The SEM instrument was equipped with a three-axis goniometer and allowed us to orient the monocrystalline $\mathrm{Au}$ (spherical) electrode and to obtain SEM images that were then superimposed on the simulated single crystal. Once superimposed with a reasonable accuracy $\left( \pm 3^{\circ}\right)$, we can identify the location of the main surface facets on the actual monocrystalline $\mathrm{Au}$ (spherical) electrode. One stereographic triangle in the actual and simulated single crystals is marked with black lines; it contains all basal, stepped, and kinked facets. It is important to add that one fcc single crystal contains 48 stereographic triangles. Figure $2 c$ shows a partially color-coded visualization of this triangle and shows as solid circles the locations of the facets which are the subject of our study. As explained above, the low Miller index surfaces are marked by red, blue, and green filled circles located at the vertices of the triangle; stepped surfaces are located along edges of the triangle. Kinked surfaces are located inside of the triangle.
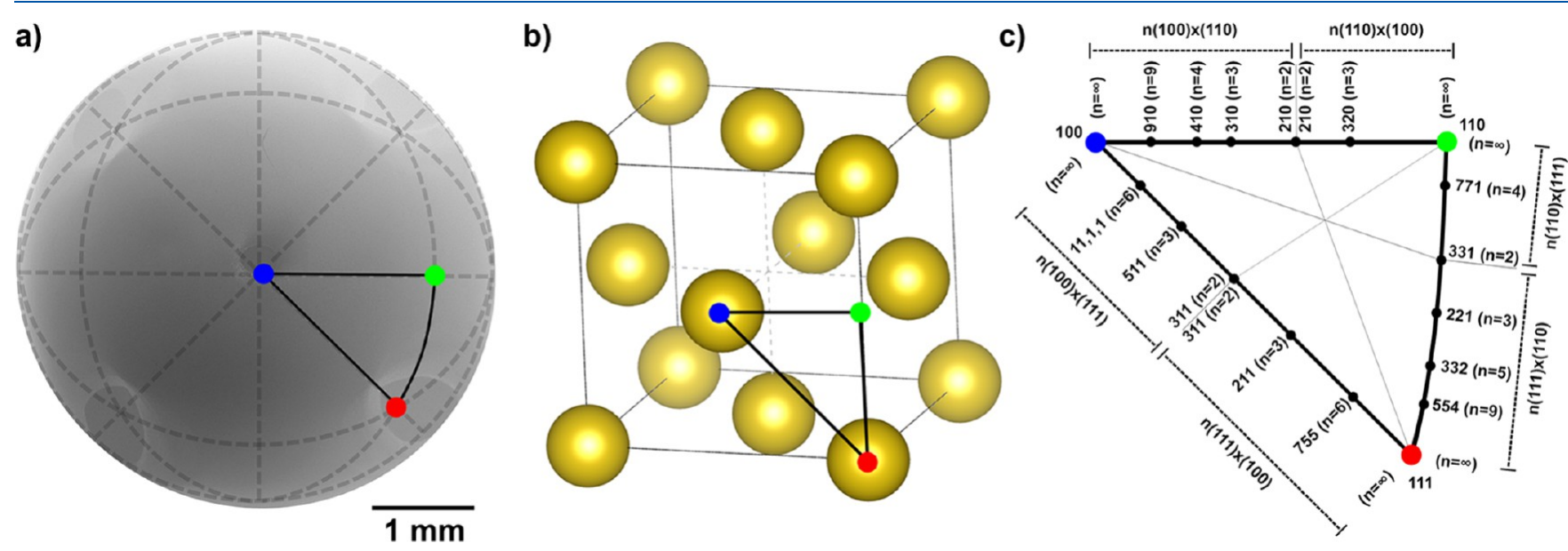

Figure 2. Methodology of the $\mathrm{Au}($ spherical) electrode and surface facet identification. (a) SEM image of an actual Au(spherical) electrode. (b) Image of a simulated spherical Au single crystal, with the low Miller index facets depicted using different colors (i.e., (111) in red, (100) in blue, and (110) in green). One stereographic triangle in these two images is marked using a black, solid line. (c) Color-coded visualization of a stereographic triangle, showing the locations of the surface structures which are the subject of our study. 


\section{RESULTS AND DISCUSSION}

3.1. Voltammetric Characterization of Cathodic Corrosion. Cathodic corrosion was investigated on single crystals oriented and cut to expose a specific facet and on spherical single crystals that were not cut and thus contain a multitude of different types of facets. The changes in the $\mathrm{CV}$ as a function of corrosion potential were investigated for the three basal planes $[\mathrm{Au}(111), \mathrm{Au}(100)$, and $\mathrm{Au}(110)]$ as well as for a spherical single crystal $[\mathrm{Au}($ sphere $)]$, where trends observed on the former will be used to interpret behavior observed on the latter.

$\mathrm{CV}$ was conducted in the $50 \mathrm{mM}$ sulfuric acid electrolyte in the range of $0<E<1.65 \mathrm{~V}_{\mathrm{RHE}}$ at a scan rate of $80 \mathrm{mV} \mathrm{s}^{-1}$ to facilitate comparison to literature data. ${ }^{15}$ Under such conditions, the gold oxidation region $\left(1.35<E<1.65 \mathrm{~V}_{\mathrm{RHE}}\right)$ exhibits a number of characteristic zones defined by the hydroxide/oxygen adsorption strength of a particular (set of) surface site(s). In this work, we focus on the gold oxidation region because the effect of introducing different types of steps at differing step densities on this region has been thoroughly studied and reported. Furthermore, changes in step density result in gradual changes in the gold oxidation region. Hence, we focus on investigating the oxidation region as opposed to the double-layer region. Although not discussed herein, closeups of the double-layer region are provided in the Supporting Information (Figure S5). The different regions, their accompanying potential window, and the way they are referred to in this work are summarized in Table 1.

Analysis of CV changes in the hydroxide/oxygen adsorption region will allow for the determination of global trends in the creation and destruction of specific types of facets. Rather than the direct conversion of one site to another, it is the formation of pits and/or nanocrystals that are responsible for the creation of specific sites, as will be discussed later in this work. We start by discussing the CV of the $\{111\}$ plane, which is depicted in Figure 3a. For this surface, the characteristic feature of an infinite $\{111\}$ terrace at $1.63 \mathrm{~V}_{\mathrm{RHE}}$ is observed to decrease in current density and shift to less positive potentials after cathodic corrosion. From the assignment of the different regions of hydroxide/oxygen adsorption on gold in Table 1, this shift is indicative of the creation of large finite-length $\{111\}$ terraces with step defects. Thus, this particular change in the $\mathrm{CV}$ denotes the introduction of step-site defects in the "infinite" $\{111\}$ plane upon corrosion. Simultaneously, the charge in the $1.46<E<1.57 \mathrm{~V}_{\mathrm{RHE}}$ region is also found to increase as more corrosive potentials are applied, which is again evidence for $\{111\}$ terraces with step defects. However, the terraces in this region are of a smaller size (i.e., a higher step density). As will be shown below, these formed stepped facets likely relate to etch pits and nanocrystallite formation on the gold surface. Finally, the imperfections that were initially present in the cut $\mathrm{Au}(111)$ surface $\left(1.35<E<1.40 \mathrm{~V}_{\mathrm{RHE}}\right.$, Table 1) exhibit nonmonotonic behavior as a function of corrosion potential. Their quantity (i.e., the charge corresponding to their features) decreases for more moderate (i.e., less negative) corrosive potentials, whereas this decrease in the number of these sites is less pronounced for more strongly corrosive potentials (e.g., moderate levels of cathodic corrosion actually result in a reduction of these types of defect sites, but this effect is less under more strongly corrosive conditions).

The voltammetry of the $\{100\}$ surface is shown in Figure $3 \mathrm{~b}$. For this facet, the characteristic feature at $1.42 \mathrm{~V}_{\mathrm{RHE}}$ is found to

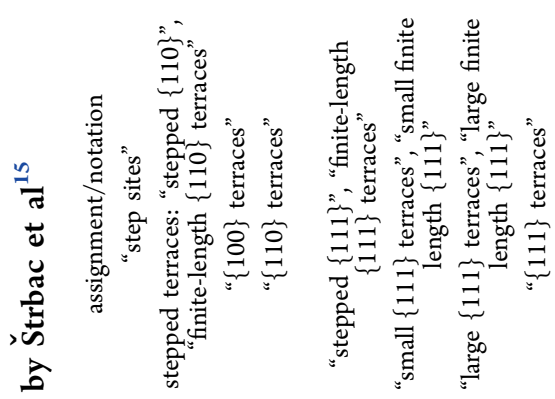

政

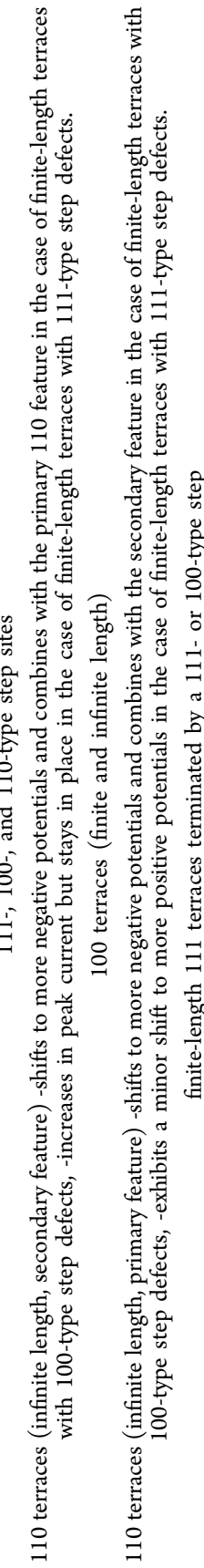

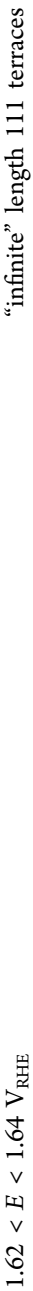




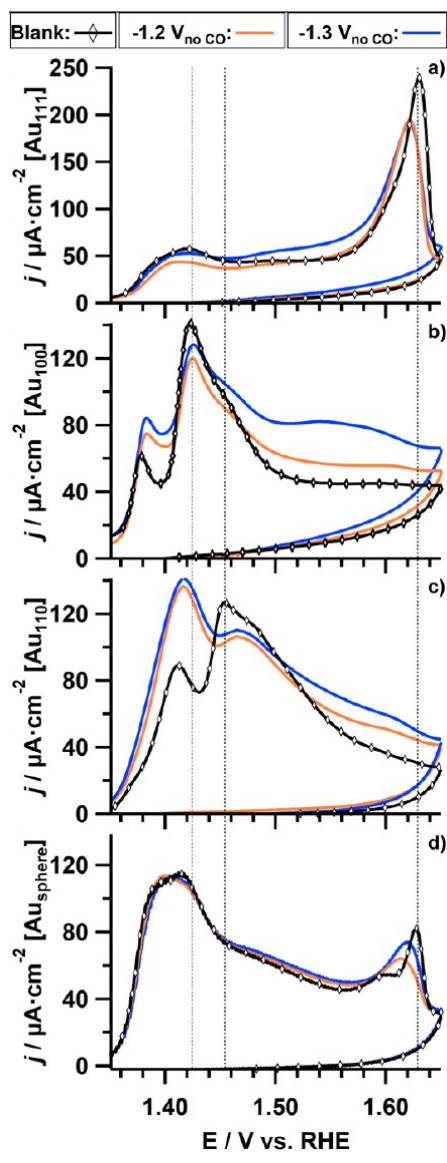

Figure 3. Cyclic voltammograms of (a) $\mathrm{Au}(111),(\mathrm{b}) \mathrm{Au}(100)$, (c) $\mathrm{Au}(110)$, and (d) $\mathrm{Au}$ (spherical) in $0.05 \mathrm{M} \mathrm{H}_{2} \mathrm{SO}_{4}$ after 2.5 min of corrosion in $10 \mathrm{M} \mathrm{NaOH}$ at $-1.2 \mathrm{~V}_{\mathrm{RHE}}$ (orange) and $-1.3 \mathrm{~V}_{\mathrm{RHE}}$ (blue) (85\% iR-corrected), compared to the blank obtained prior to corrosion (black with diamonds).

initially slightly decrease in peak intensity upon corrosion. However, surfaces corroded more vigorously (i.e., having been subjected to increasingly negative potentials) exhibit less of a decrease in the number of $\{100\}$ terraces than surfaces corroded under milder conditions, although the peak is still lower than that for an uncorroded surface. The first observation suggests that under mild cathodic potentials (with respect to the onset of cathodic corrosion, $-0.8 \mathrm{~V}$ ), ${ }^{20}$ $\{100\}$ terrace sites are transformed and/or destroyed. The second observation can be explained by taking into account that finite-length $\{100\}$ terraces give rise to a broad contribution in the same region as infinite $\{100\}$ terraces, the two eventually being indistinguishable from one another. Thus, although we corrode our initially "perfect" 100 plane, it is still possible to observe a gain in charge in the $\{100\}$-terracecharacteristic potential window if we assume that corrosion leads to the creation of finite-length $\{100\}$ terraces. Corrosion of the $\{100\}$ terraces is further corroborated by an increase of step-site defects (feature at $1.38 \mathrm{~V}_{\mathrm{RHE}}$, Table 1) as increasingly cathodic potentials are applied. Finally, it is observed that corrosion of the $\{100\}$ plane leads to a substantial increase in the number of stepped $\{111\}$ terrace sites, as denoted by an increase in charge in the $1.46<E<1.62 \mathrm{~V}_{\mathrm{RHE}}$ region. Apparently, corrosion introduces specifically $\{111\}$-type step defects into the $\{100\}$ surface and thereby transitions the overall surface morphology in the direction of the $\{111\}$ plane.
Corrosion behavior of the most open of the basal planes, $\{110\}$, is shown in Figure 3c. Unlike the other two planes, this facet exhibits two characteristic features in the case of infinite terrace lengths: a weaker feature at $1.41 \mathrm{~V}_{\mathrm{RHE}}$ (denoted from here on as secondary) and a stronger one at $1.45 \mathrm{~V}_{\mathrm{RHE}}$ (which we shall refer to as primary). Under corrosive conditions, the primary feature decreases in current density. However, similar to what is observed for corrosion of the $\{100\}$ plane, this decrease in peak current density is actually less when more corrosive potentials are applied. As for the secondary feature, it is found to monotonically increase with corrosion potential instead, without shifting. Finally, an increase in charge is observed for the region characteristic for finite-length stepped $\{111\}$ terraces. Taken together, these observations can only be explained by the transformation of the $\{110\}$ plane into a surface that is rich in $\{111\}$-type sites. The fact that the primary $\{110\}$ peak exhibits less of a decrease in current density as more corrosive potentials are applied can be explained by the fact that finite-length $\{111\}$ terraces contribute charge in a very broad potential range. Although the number of $\{110\}$ terrace sites is expected to decrease more at stronger corrosive potentials, the contribution of charge by finite-length $\{111\}$ terraces increases.

Figure $3 \mathrm{~d}$ illustrates the transformation of the $\mathrm{CV}$ of a spherical single crystal upon cathodic corrosion. Overall, the changes in the CV of a spherical single crystal appear less pronounced than the changes for the other (cut) single-crystal facets. Notwithstanding, the following changes are observed upon cathodic corrosion: the characteristic feature of the infinitely large $\{111\}$ terrace sites at $1.63 \mathrm{~V}_{\mathrm{RHE}}$ is observed to shift to less positive potentials, where it is presumed to (partially) merge with the voltammetric feature characteristic of large finite-width stepped $\{111\}$ terraces, leading to the maximum of the latter feature shifting to more positive potentials. Furthermore, an increase in charge for smaller stepped $\{111\}$ terraces is observed. This behavior mimics what is observed upon corrosion of the $\{111\}$ electrode and can be explained by the creation of step-site defects, leading to finitelength stepped $\{111\}$ terraces with decreasing terrace lengths as the surface corrodes further.

Finally, small changes are observed in the (stepped) $\{100\}$ terrace and step- and kink-site regions (Table 1$)$. This "twin peak" feature merges into a single peak, only showing minor changes in peak current without a clear trend. More specifically, depending on corrosion conditions (not depicted), its current density can decrease or increase marginally. Such erratic behavior is in line with the behavior observed for corrosion of $\mathrm{Au}(111)$ specifically, where the charge for stepsite defects could increase as well as decrease, depending on experimental conditions. Although such inconsistent behavior was not observed upon corrosion of the $\{100\}$ plane, it can still be reasonably explained by $\{111\}$ being the dominant facet on the gold spherical crystal because of them adopting the equilibrium crystal shape during growth (see Figure $2 a){ }^{27}$

Because voltammetry for stepped $\{111\}$ terraces is ambiguous (100- and 111-type steps cannot be clearly distinguished via hydroxide/oxygen adsorption in the CV profile), the voltammetry measurements cannot provide direct evidence for the types of steps that are formed upon corrosion of the $\{111\}$ plane; it might be preferentially one step type or both step types.

3.2. Morphological Characterization of Cathodic Corrosion. 3.2.1. SEM Anisotropy of the Basal Planes. 


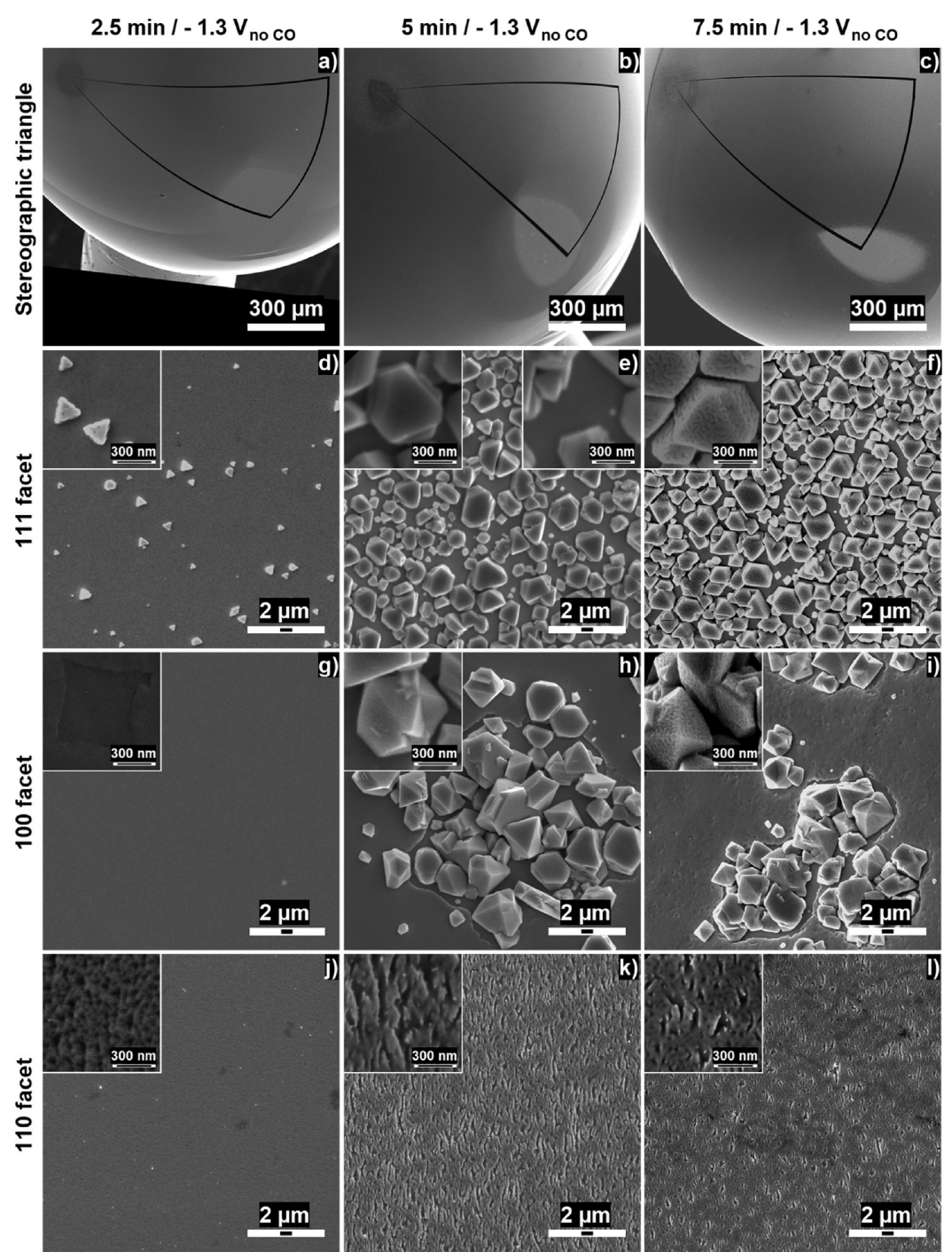

Figure 4. SEM images (macroscopic overview, the 111 facet, 100 facet, and 110 facet, going from the first row to the last row) of anisotropic surface corrosion in the form of pitting and nanocrystal formation as a function of time $(2.5-7.5 \mathrm{~min}$, going from the left column to the right column) at a corrosion potential of $-1.3 \mathrm{~V}_{\mathrm{RHE}}(85 \%$ iR-corrected) in $10 \mathrm{M} \mathrm{NaOH}$.

Further information regarding the facet dependency of cathodic corrosion is derived from SEM images. Because of the $\mathrm{Au}$ spherical single crystal adopting the fcc equilibrium crystal shape, it is possible to determine the locations of all facets so long as one can accurately pinpoint a minimum of two facets and knows the radius of the crystal. Under the effects of corrosion, finding two identification points is straightforward, as can be seen in Figure $4 a-c$. From this figure, which depicts a global overview of spherical singlecrystal electrodes corroded for different time intervals at -1.3 $V_{\text {RHE }}$, it can be seen that the $\{100\}$ and $\{111\}$ facets are easily identified.

The anisotropy in corrosion that was identified from $\mathrm{CV}$ experiments is also observed macroscopically in the SEM images. This can be seen in the form of differences in timedependent evolution of the different basal planes as well as in the differences in contrast for different locations on the surface. However, the anisotropic behavior is much clearer in the images shown in Figure $4 d-1$, where close-ups of the three basal planes are shown. More specifically, Figure $4 d-f$ consists of higher-magnification images of the $\{111\}$ plane corroded for $2.5,5$, and $7.5 \mathrm{~min}$ at $-1.3 \mathrm{~V}_{\mathrm{RHE}}$ in $10 \mathrm{M} \mathrm{NaOH}$, respectively, whereas Figure $4 \mathrm{~g}-\mathrm{i}, \mathrm{j}-\mathrm{l}$ are SEM images of the $\{100\}$ and $\{110\}$ faces under the same conditions.

From these expanded images, it can be seen that the morphological changes of the basal planes as a function of corrosion time differ per facet. The initially flat $\{111\}$ face shows only very minor signs of pitting and etching but does exhibit nanocrystal formation when certain corrosion potential and time thresholds are exceeded (specifically, $-1.2 \mathrm{~V}_{\mathrm{RHE}}$ for $\geq 7.5 \mathrm{~min}$, Figure S1a,b). Of course, we realize that SEM cannot image truly nanoscopic changes on the surface. Specifically, uniformly distributed nanocrystals with a clear preference for a triangular prism and octahedral geometry start to form (Figure 4d,e), with increasing crystallite density as a function of corrosion time. Although pitting of the flat face is observed (Figure S2a) after the onset of nanocrystal formation, these newly formed nanocrystals themselves exhibit much more prominent pitting (Figure $4 \mathrm{e}, \mathrm{f}$ ).

Compared to the $\{111\}$ plane, only minor signs of surface pitting are observed for the $\{100\}$ facet prior to the onset of nanocrystal formation. The earliest observed signs of pitting can be seen in Figure $4 \mathrm{~g}$ (showing an uncommonly observed pit) but become more pronounced when nanocrystals start to 


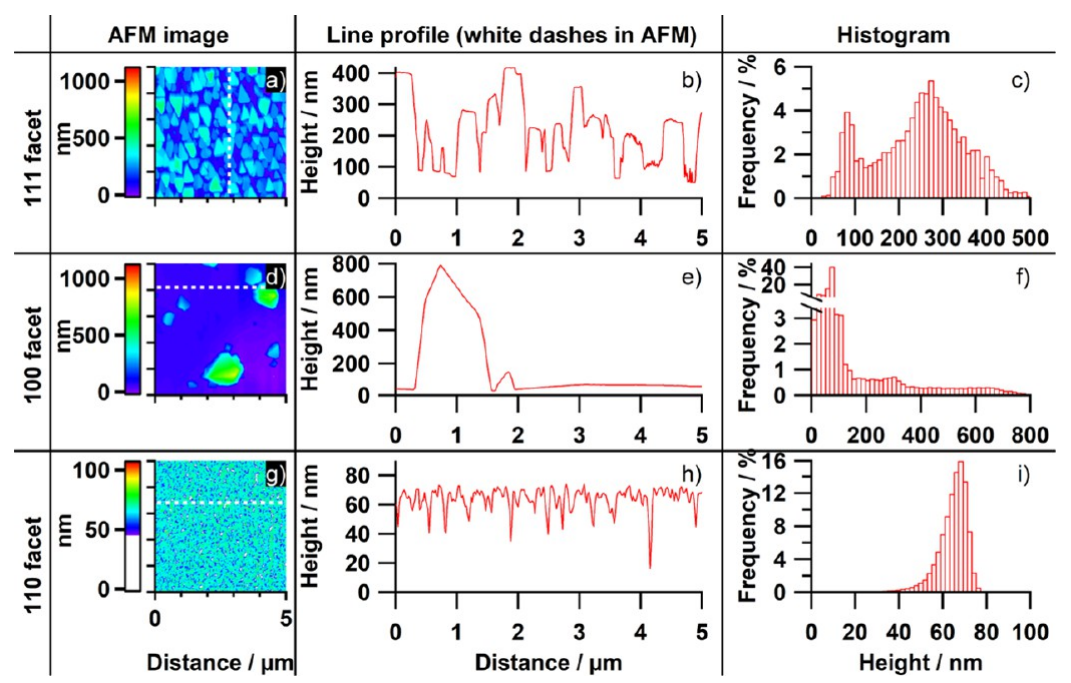

Figure 5. AFM images, line profiles, and histograms (columns 1, 2, and 3, respectively) for the basal planes $[\{111\}(a-c),\{100\}(d-f)$, and $\{110\}$ $(\mathrm{g}-\mathrm{i})]$ after cathodic corrosion at $-1.3 \mathrm{~V}_{\mathrm{RHE}}(85 \% \mathrm{iR}$-corrected) for $5 \mathrm{~min}$ in $10 \mathrm{M} \mathrm{NaOH}$.

grow (Figure S2b). Again, under sufficiently cathodic conditions, nanocrystals are also observed on the "infinite" $\{100\}$ plane (specifically, $-1.3 \mathrm{~V}_{\mathrm{RHE}}$ for $\geq 5 \mathrm{~min}$, compare Figure $4 \mathrm{~g}-\mathrm{h}$ ) and these nanocrystals themselves are also susceptible to surface pitting (Figure 4i, inset). However, some differences exist when comparing nanocrystal formation on $\{100\}$ and $\{111\}$. First, the onset of nanocrystal formation is shifted to more cathodic potentials. Second, rather than depositing uniformly, nanocrystals on the $\{100\}$ facet tend to cluster together (compare Figure $4 \mathrm{e}, \mathrm{h}$ ). Third, whereas the nanocrystals that deposit on the $\{111\}$ face preferentially adopt a triangular-prism geometry (with the word prism denoting the top face to be parallel with and equal in shape to the base, similar to a column), the (nano)crystallites that form on the $\{100\}$ face are better described as adopting shapes driven by a truncation process (where truncation refers to the (partial) cutting of the vertices of a polyhedron, resulting in the creation new faces in their stead) and/or aggregates of crystallites, with some of the smaller individual crystallites adopting an octahedral shape.

Cathodic corrosion-induced morphological changes for the $\{110\}$ plane do not follow what is observed for the $\{111\}$ and $\{100\}$ planes. As opposed to mild surface pitting paired with nanocrystal deposition under sufficiently corrosive conditions, severe surface pitting takes place without the formation of nanocrystals (see e.g., Figure 41). Corrosion behavior of this facet is dominated by surface etching and pitting, as can be seen in Figure $4 \mathrm{j}-\mathrm{l}$. The onset of pitting in the $\{110\}$ face as observed in SEM images $\left(-1.1 \mathrm{~V}_{\mathrm{RHE}}\right.$ for $\geq 5 \mathrm{~min}$, Figure $\left.\mathrm{S} 1 \mathrm{c}, \mathrm{d}\right)$ occurs earlier than SEM-observable morphological changes (e.g., pitting, nanocrystal formation) for the $\{111\}$ and $\{100\}$ faces, with the earliest signs of corrosion of the latter two faces requiring potentials equal to or more negative than $-1.2 \mathrm{~V}_{\mathrm{RHE}}$ at corrosion times exceeding 5 min.

The observed nanocrystal growth is found to depend on both corrosion time and corrosion potential. In Figure $4 \mathrm{~d}-\mathrm{f}$, the corrosion time is varied for the same applied potential, showing a clear increase in nanocrystal density with respect to time. The dependency of nanocrystal formation on potential is evident when comparing the onset potential for nanocrystal formation on the $\{111\}$ face as discussed previously $(-1.2$ $\mathrm{V}_{\mathrm{RHE}}$ for $\geq 7.5 \mathrm{~min}$, Figure $\mathrm{S} 1 \mathrm{a}, \mathrm{b}$ ) with the onset of nanocrystal formation at an applied potential of $-1.3 \mathrm{~V}_{\mathrm{RHE}}: \geq 2.5 \mathrm{~min}$ (Figure 4d). This appears to suggest that nanocrystal formation depends on the buildup of a concentration gradient of dissolved gold ions, originating from surface etching under cathodic potentials. More cathodic potentials lead to faster dissolution, resulting in reaching supersaturation values sooner and thus an earlier onset of nanocrystal formation. However, the facets that exhibit nanocrystal deposition during corrosion experiments actually show little surface pitting prior to (and arguably after) the onset of nanocrystal formation. Rather than the flat basal planes being the origin of the gold ions, we hypothesize the gold ions to originate from the dissolution of facets with step defects as we expect them to be more corrosive.

3.2.2. AFM Anisotropy of the Basal Planes. Further insight into surface dissolution and ion redeposition phenomena is derived from AFM imaging, as shown for the three basal planes in Figure 5. First, in Figure $5 \mathrm{a}-\mathrm{c}$, a $5 \times 5 \mu \mathrm{m}$ AFM image and the accompanying line profile and histogram for a corroded $\mathrm{Au}(111)$ surface are depicted. The AFM image agrees with SEM data (Figure 4e), showing nanocrystal growth with uniformly distributed nanocrystals with a preferential triangular-prism geometry. From the line profile (Figure 5b), it becomes evident that these nanocrystals have fairly flat tops that run parallel to the substrate on which they grow. Furthermore, the spots where nanocrystals are absent (purple-blue-colored regions in Figure 5a) show little variation in height. Hence, the majority of the roughening occurring for this facet stems from the growth of nanocrystals rather than from dissolution/corrosion of the surface itself. This also agrees with previously discussed SEM data, where parts of the surface that do not contain crystallites appear to be "smooth".

An AFM image of the $\{100\}$ plane, with the accompanying line profile and histogram, can be seen in Figure $5 \mathrm{~d}-\mathrm{f}$, respectively. Contrary to the behavior of nanocrystal growth on the $\{111\}$ plane, nanocrystals that grow on the $\{100\}$ plane have the tendency to form clusters of crystallites, resulting in nonuniform surface coverage. Furthermore, the clustering results in nanocrystals that have seemingly arbitrary shapes, although smaller individual crystallites exhibit preference for an octahedral geometry, as observed from both SEM and AFM. Contrary to the smoothly terminated "plateau like" nanocryst- 


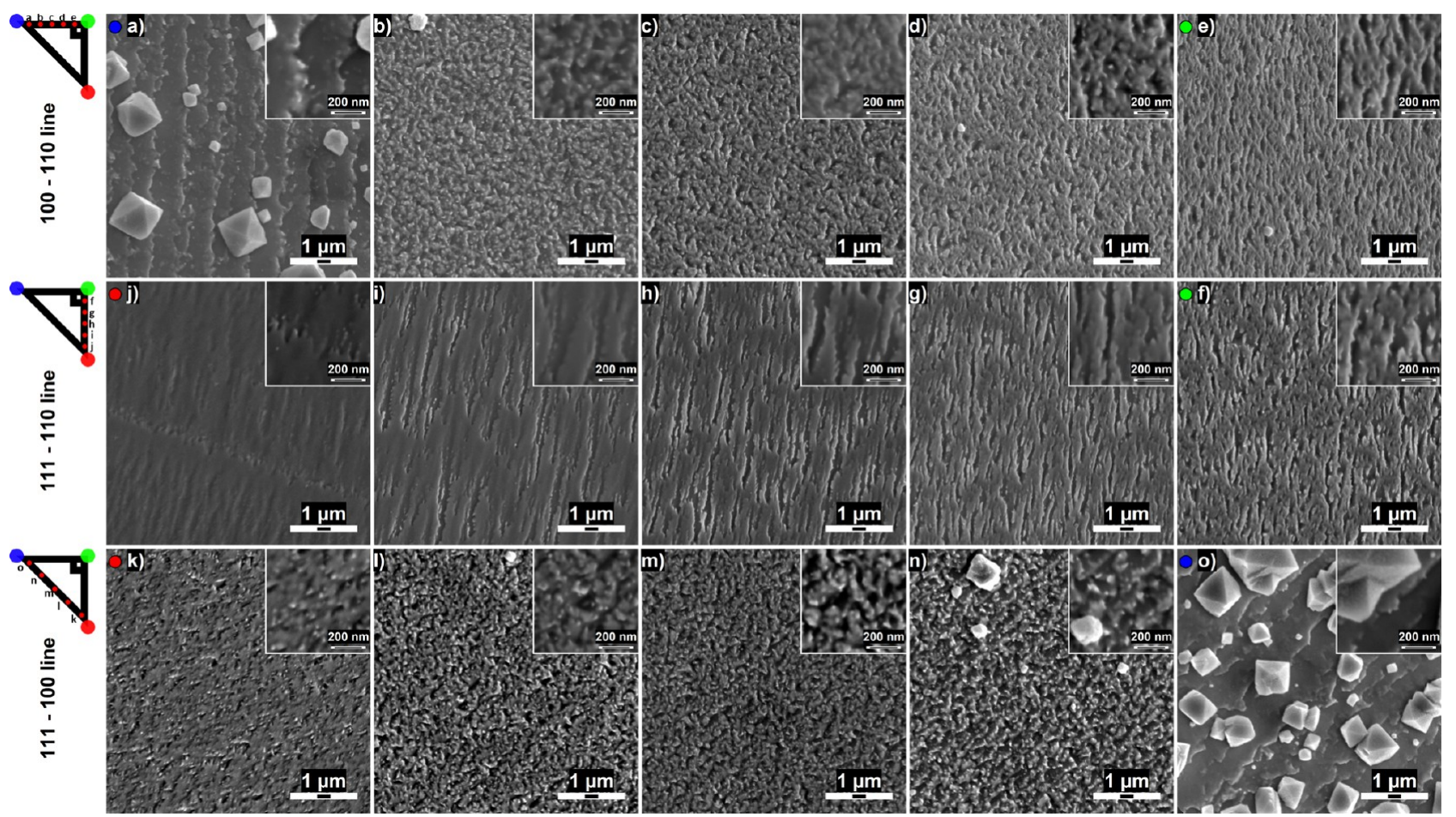

Figure 6. Effect of step type and step density on macroscopic corrosion phenomena at $-1.3 \mathrm{~V}_{\mathrm{RHE}}$ ( $85 \%$ iR-corrected) for $5 \mathrm{~min}$, as investigated by traversing the stereographic triangle in its entirety.

als that form on the $\{111\}$ face, the nanocrystals that grow on the $\{100\}$ face are better described as "mountainous". As for corrosion of the plane underneath the nanocrystals, a fairly smooth surface is obtained, which is in line with what is observed for corrosion of $\mathrm{Au}(111)$.

The final facet, $\mathrm{Au}(110)$, is shown in Figure $5 \mathrm{~g}-\mathrm{i}$, containing, respectively, an AFM image and the accompanying line profile and histogram. For this plane, no nanocrystal formation is observed upon corrosion, which is in line with what SEM data show (Figure 4k). However, the surface clearly exhibits pitting, with these pits being distributed uniformly across the surface and reaching depths of several tens of nanometers.

3.3. Enhanced Corrosion at Stepped Surfaces. Because of the regularity of spherical single crystals, facets with stepand kink-site defects are located between the basal planes on the stereographic triangle, with the step density increasing as one moves further away from any of the three basal planes. As such, it is possible to investigate the surface morphology after corrosion as a function of step type and step density by imaging different parts of the three sides of the stereographic triangle as defined by the $\{111\},\{100\}$, and $\{110\}$ faces. The results of such an experiment are shown in Figure 6 (identical corrosion conditions as in Figure 4e,h,k), wherein we traverse along the edges of the stereographic triangle and take an image of the surface at equidistant intervals, varying step density and transitioning step types along the way.

3.3.1. Corrosive Effect of Introducing Minor Amounts of Defects in the $\{100\}$ and $\{111\}$ Planes. The effect of incorporating a small number of step-site defects (i.e., low step density) into "infinitely large" (unstepped) $\{100\}$ and $\{111\}$ terraces can be investigated by comparing Figure $4 \mathrm{~h}$ ("perfect" $\{100\})$ with Figure 6a,o $(n\{100\} \times\{110\}$ and $(n\{100\} \times\{111\})$ and by comparing Figure 4e ("perfect" $\{111\})$ with Figure 6j,k $(n\{111\} \times\{110\}$ and $n\{111\} \times\{100\})$.
For this comparison, we shall neglect nanocrystal growth on the infinite terraces and focus on the areas in the SEM images that do not contain nanocrystals (e.g., in between crystallites such as in the right inset in Figure 4e and/or nanocrystal-free patches, such as the corners in Figure $4 \mathrm{~h})$. From these images, it is found that the infinite $\{100\}$ and $\{111\}$ faces yield much smoother surfaces post corrosion as compared to the same surfaces with a minor density of step-site defects, which yield visually coarser surfaces upon corrosion (mind the two-fold difference in magnification between Figures 4 and 6). Because the presence of small amounts of step-site defects results in visually more roughened surfaces upon corrosion and because of the fact that these facets with a low step density are located in close spatial proximity to their unstepped counterparts, we hypothesize that dissolution of gold atoms at these stepped facets drives the concentration gradient that we think is responsible for nanocrystal formation as observed on the "infinite" $\{111\}$ and $\{100\}$ faces.

3.3.2. Corrosive Effect of Varying the Density of $\{110\}-$ Type Steps in the $\{100\}$ and $\{111\}$ Planes. We start the investigation into the effect of step type and step density on corrosion behavior by looking at the effect of $\{110\}$ step-site defects terminating $\{100\}$ and $\{111\}$ terraces (Figure $6 \mathrm{a}-\mathrm{c}, \mathrm{h}-$ $j$, respectively). From these images, it can be seen that surfaces that contain $\{110\}$ step-site defects are highly susceptible toward cathodic corrosion. Interestingly, the mode of corrosion in the presence of this particular step type appears to depend on the neighboring terrace site: $\{100\}$ or $\{111\}$. Corrosion of $\{100\}$ terraces next to $\{110\}$-type steps with a low step density (Figure 6a) results in a "staircaselike" surface with fairly straight step edges on which nanocrystallites grow. However, increasing the number of step-site defects (Figure $6 \mathrm{~b}, \mathrm{c})$ yields a much coarser surface exhibiting uniform microroughening without nanocrystal growth during corrosion. Quite different morphological changes are observed when 
$\{111\}$ terraces with $\{110\}$-type step defects undergo cathodic corrosion: first, both low (Figure 6j) and higher (Figure 6h,i) step densities result in a similar type of surface. Second, corrosion of the steps is found to yield densely packed trenches that are open-ended on one side (Figure 6h,i) with the number of trenches per unit area diminishing as step density is reduced (Figure 6j). Overall, the surface morphology after corrosion becomes "smoother" for the facets with fewer step-site defects, leading to the observation that the $\{111\}$ terraces by themselves are less corrosive than when terminated by $\{110\}$-type step defects. This observed difference between corrosion of $\{111\}$ facets and $\{100\}$ facets in the presence of $\{110\}$-type steps may, in part, originate from redeposition phenomena. Specifically, the onset of nanocrystal formation on the $\{100\}$ plane is observed to initiate at the edges of this face, where the terraces with low-density step defects are located (Figure S3a,b) before they start to concentrate on the defectfree facet, as opposed to crystallite formation on the $\{111\}$ plane (see e.g., Figure S1a,b), where nanocrystals are only observed on the defect-free face.

3.3.3. Corrosive Effect of Varying the Density of $\{100\}-$ and $\{111\}$-Type Steps in the $\{110\}$ Plane. The presence of $\{100\}$ and $\{111\}$ types of step defects in $\{110\}$ terraces is found to have much less of an increasing corrosive effect than the presence of $\{110\}$-type step defects in $\{111\}$ or $\{100\}$ terraces. Specifically, in the case of $\{100\}$-type step defects in $\{110\}$ terraces with low (Figure 6e) and higher (Figure 6c,d) step densities, it is found that step and terrace sites cannot be distinguished in the SEM images and that the surface morphology remains virtually identical as step density increases. This, combined with the strong resemblance of the morphology of these surfaces with the morphology of corroded $\{110\}$ terraces without step-site defects (Figure 4k), indicates that post-corrosion morphology is dominated by corrosion of the $\{110\}$ terrace sites and indifferent from the presence of $\{100\}$ step sites.

Corrosion of $\{110\}$ terraces in the presence of $\{111\}$-type steps of low (Figure 6f) and higher (Figure 6g,h) densities exhibits behavior comparable to the presence of $\{100\}$-type steps, yielding in a surface containing many pits that are a few hundred nanometers in size. However, unlike $\{100\}$ step sites, increasing the density of $\{111\}$-type steps (going from Figure $6 \mathrm{f}-\mathrm{h}$ ) does lead to morphological changes. Specifically, the pits become more elongated to yield trenches, which in turn become seemingly open-ended on one side as the step density is further increased. Rather than stating that these morphological changes originate from the presence of increased amounts of $\{111\}$-type steps, it is more accurate to state that corrosion of the $\{110\}$ terraces transitions into corrosion of $\{110\}$-type step sites. This reasoning can be substantiated by comparing the facets we just discussed to the facets located closer to the $\{111\}$ basal plane along the same stereographic line (Figure 6i,j). These latter images are of corroded $\{111\}$ terraces with differing amounts of $\{110\}$-type step defects and exhibit the same types of trenches as the corroded $\{110\}$ terraces with $\{111\}$-type step defects. As the trenches decrease as we get closer to the $\{111\}$ plane, the $\{110\}$-type steps yield the trenchlike corrosion feature as opposed to corrosion of $\{111\}$-type steps. Therefore, the changes in surface morphology upon increasing the step density of $\{111\}$-type defects in $\{110\}$ terraces are better described by stating that the corrosion behavior of $\{110\}$ terraces evolves to resemble the corrosion of $\{110\}$-type steps as terrace width is decreased.
3.3.4. Corrosive Effect of Varying the Density of \{100\}Type Steps in the $\{111\}$ Plane and $\{111\}$-Type Steps in the $\{100\}$ Plane. Corrosion of $\{111\}$ terraces in the presence of low (Figure 6k) and higher (Figure 6l,m) densities of $\{100\}$ type steps yields a morphology that is quite different from corrosion in the presence of $\{110\}$-type steps. Rather than a relatively smooth surface with trenches, a uniformly roughened surface is obtained instead. Furthermore, the surface morphology does not change much when the density of step-site defects is increased (going from 6k to $\mathrm{m}$ ). As such, it is concluded that morphology is dictated by corrosion of the $(\{111\})$ terrace sites rather than by corrosion of the $\{100\}$ step sites.

The final effect that can be studied from these images is the corrosion behavior of $\{100\}$ terraces with lower (Figure 6o) or higher (Figure $6 \mathrm{~m}, \mathrm{n}$ ) densities of $\{111\}$-type step defects. Corrosion of terraces with high amounts of step defects yields a morphology that resembles corrosion of $\{111\}$ terraces, whereas lowering the step-site density further (Figure 6o) results once more into a "staircaselike" morphology with fairly straight edges and crystallite formation, as was also observed in the case of corrosion in the presence of low-step-density $\{110\}$-type step defects. Hence, the morphology is dominated by corrosion of step-site defects rather than the $\{100\}$ terraces.

Summarizing, corrosion resistivity of the three basal planes and their stepped counterparts is found to increase in the following order

$$
\begin{aligned}
& \{110\} \text { terraces } \approx \text { stepped }\{110\} \text { terraces } \\
& \quad<\text { terraces with }\{110\} \text { steps }<\text { other stepped terraces } \\
& \quad<\{111\} \text { terraces }<\{100\} \text { terraces }
\end{aligned}
$$

where

- The presence of any type of $\{110\}$ site leads to the formation of preferentially oriented trenches, which is more pronounced in the case of steps.

- Stepped $\{111\}$ terraces exhibit homogeneous microscale roughening in the presence of $\{100\}$ steps, compared to trench formation in the presence of $\{110\}$ steps.

- Stepped $\{100\}$ terraces exhibit uniform roughening at higher step densities and a "staircaselike" morphology with nanocrystal growth at lower step densities.

- "Infinite" $\{111\}$ and $\{100\}$ basal planes exhibit the highest corrosion resistance with the latter showing a later onset for nanocrystal formation and surface pitting.

3.4. Location, Shapes, and Origin of Nanocrystallites. In the preceding sections, we have shown that facet-specific morphological changes occur during cathodic corrosion of gold. Specifically, the $\{110\}$ face and facets with higher Miller indices exhibit cathodically induced surface dissolution resulting in pitting, whereas the $\{111\}$ and $\{100\}$ faces mostly show nanocrystallite growth which, we hypothesize, originates from the gold that dissolves at the more corrosive facets. Dissolution from the only other possible source of gold, the counter electrode, was excluded based on the following: first, nanoparticle formation on gold during cathodic corrosion has been reported in the literature before in a work in which a titanium counter electrode was used, with crystallite shapes matching what is observed in this work. ${ }^{20}$ Second, similar crystallite growth was observed over the entire crystal both for the faces pointing toward the counter electrode and for the faces on the opposite side of the crystal. The nonuniform 
concentration gradient that would result from dissolution of the counter electrode would most likely not allow for such symmetry. Therefore, there is no effect of the placement of the counter electrode on the surface modification. Third, although it is known that gold dissolution takes place at anodic potentials, the majority of the dissolution actually occurs in the reducing cycle rather than during the anodic cycle. ${ }^{28,29}$ As a DC signal was applied during measurements, no such cycling occurred on the counter electrode. Furthermore, gold is known to exhibit a decrease in dissolution rate (passivation) as increasingly positive potentials are applied (with commonly observed counter electrode potentials in the $+3 \mathrm{~V}_{\mathrm{RHE}}$ range in this work). ${ }^{28}$ Finally, cathodic corrosion for much longer times $(1.5 \mathrm{~h})$ at a slightly lower cathodic potential $\left(-1 \mathrm{~V}_{\mathrm{RHE}}\right)$ in the presence of $\mathrm{CO}$ did not yield any nanoparticle formation, even though the counter electrode for this experiment was still at ca. $2.5 \mathrm{~V}_{\mathrm{RHE}}$ (Figure S4).

Surface dissolution is commonly observed under cathodic conditions for various noble-metal surfaces $9,11,20,21,30$ and is hypothesized to occur via the formation of a negatively charged ternary metal hydride complex..$^{9,11,31,32}$ Interestingly, for platinum $^{33}$ and rhodium, ${ }^{20}$ solution-phase nanoparticles are observed under sufficiently high electrolyte conditions and at sufficiently negative potentials, where agglomeration and deposition of these solution-phase formed particles back onto the substrate are hypothesized to be the source of the nanoparticles that are (sometimes) observed on these surfaces after cathodic corrosion. ${ }^{9}$ However, in this work, no indications for the formation of such solution-phase particles (in the form of a color change of the electrolyte, the formation of a "particle cloud" during corrosion, or the formation of a precipitate after cathodic corrosion) have been observed even at applied potentials as negative as $-1.3 \mathrm{~V}$. However, nanocrystallite growth is observed. Interestingly, these crystallites grow only on facets of specific orientations $(\{111\}$ and $\{100\})$, with the underlying surface furthermore having a "templating effect", where it influences the shapes of the crystallites that grow thereon.

It has been reported that for shape manipulation of gold nanoparticles, there exist two distinct mechanisms. ${ }^{34}$ First, one can control the rate at which a dissolved precursor ion is converted to the solid state, which is called "kinetic control". Second, one distinguishes selective surface passivation, whereby the growth of a particular facet is retarded because of it being blocked by some adsorbing species. In the case of kinetic control, thermodynamically optimal shapes are obtained for infinitely slow growth rates, with less thermodynamically stable particles formed at increased growth rates. In the case of selective surface passivation, the eventual particle shape will be such that its surface is entirely composed of the facet that is being blocked.

If the crystallites that we observe herein were directed by selective surface passivation, their shapes should be similar/ equal everywhere as the electrolyte contains the same possible adsorbates everywhere. Furthermore, under such circumstances, no substrate directing effects would be expected but are observed. Hence, the shapes of the formed nanocrystallites must be under kinetic control instead. This is plausible if our assumption of the origin of the solution-phase gold ions is correct (i.e., stemming from dissolution of more corrosive facets) as then the concentration of gold ions is locationspecific.
In the case of kinetic control, particles with truncated bitetrahedral (e.g., triangular prism-like) and octahedral shapes are expected for slower growth rates, whereas more complex shapes (i.e., containing more edges, having lower symmetry) are expected in the case of faster growth. Slow-growth-rate particle shapes agree well with the shapes observed in this work for the nanocrystallites that grow on the $\{111\}$ face, validating our assumption that growth is under kinetic control. However, the shapes of the nanocrystallites that form on the $\{100\}$ face exhibit a plethora of differing geometries which are difficult to reconcile with a specific expected particle shape (although some of the smaller, individual ones adopt an octahedral geometry). We think that the following discussion can reasonably explain such irregular shapes as observed for the $\{100\}$ face.

The onset for crystallite growth is different on the $\{111\}$ and $\{100\}$ faces, with the $\{111\}$ face exhibiting nanocrystal formation much prior to crystallite growth occurring on the $\{100\}$ face. As no typical signs of solution-phase nanoparticle formation were observed, we hypothesize that seed formation takes place on the substrate, with seeds forming (and growing) earlier on the $\{111\}$ facet than on the $\{100\}$ facet. If a seed that forms on the substrate adopts the underlying substrate structure, then seeds that form and grow on the $\{111\}$ face are thermodynamically favorable as this facet is most stable in the case of gold. We hypothesize that this would result in a lower barrier for nucleation and would thus require a lower ion concentration, which is observed in the form of an earlier onset in nanocrystallite formation on the $\{111\}$ face, with crystallite shapes matching expected shapes for particles under kinetic control with a slow growth rate. However, as seeds forming on the $\{100\}$ face initially adopt the $\{100\}$ geometry, their nucleation is less favorable than that for the $\{111\}$ geometry, leading to a higher barrier for their formation. This translates into requiring a larger driving force in the form of a higher ion concentration. Additionally, the seed adopts the $\{100\}$ geometry while the thermodynamically favored facet is of the $\{111\}$ geometry, resulting in slower growth of these seeds. As seed formation and propagation is slower, a larger concentration gradient is formed. As this translates into a higher driving force, more unfavorable particle shapes become thermodynamically possible. As a certain threshold concentration is reached, nucleation and growth barriers are overcome and growth rates increase rapidly, resulting in irregular nanocrystallites with nonequilibrium shapes.

As for why only nanocrystallite formation and growth is observed on the $\{111\}$ and $\{100\}$ faces, a simple stability argument can be used. Under cathodic conditions, it is evident that the $\{110\}$ face and facets with higher Miller indices are unstable as they are observed to strongly corrode cathodically. Only the $\{111\}$ and $\{100\}$ faces are (relatively) stable, and hence, only seeds of these orientations are thermodynamically viable. Thus, nanocrystallites only form on these faces and not on any others. Hence, for the nanocrystallites in this work to form where they do (and do not), two conditions have to be met. First, seed nucleation must take place only on the substrate (where its orientation is influenced by the substrate) and not in the solution. Second, a sufficiently high concentration of solution-phase gold ions must be present and/or be allowed to build up.

3.5. Effect of Carbon Monoxide on Cathodic Corrosion. 3.5.1. Voltammetric Characterization of the $\mathrm{CO}$ Effect on Cathodic Corrosion. To investigate the effect 
that $\mathrm{CO}_{2}$ reduction taking place on gold would have on cathodic corrosion in a controlled setting, the following experiment was conducted: cathodic corrosion was carried out in $10 \mathrm{M} \mathrm{NaOH}$ to facilitate comparison with prior cathodic corrosion literature studies as well as to mimic the high local alkalinity expected for $\mathrm{CO}_{2}$ electrolyzers operated at high current densities. ${ }^{35}$ As gold is a good catalyst with a high Faradaic efficiency and activity for the $\mathrm{CO}_{2} \mathrm{RR}$ to $\mathrm{CO}$, the concentration of $\mathrm{CO}$ near the surface will be high. Hence, $\mathrm{CO}_{2} \mathrm{RR}$ conditions were approximated by addition of gaseous $\mathrm{CO}$ to the cell during corrosion experiments. With these settings, local $\mathrm{pH}$ effects on cathodic corrosion can be excluded as $\mathrm{pH}$ changes can be neglected because of the high starting $\mathrm{pH}$. Additionally, the continuous purging of the electrolyte with $\mathrm{CO}$ (which is not further reduced on gold) yields a constant concentration of carbon monoxide near the surface that is independent of current density. Although fixing the electrolyte $\mathrm{pH}$ allows one to determine solely the effect of the presence of $\mathrm{CO}$ on catalyst stability at an interface that is mostly independent on current density, this comes at the cost of neglecting any (possible) additional effects of the presence of $\mathrm{CO}_{2}(\mathrm{aq})$ and (bi)carbonate salts in the electrolyte. Therefore, any observed changes in the presence of carbon monoxide reflect only a part of the stability differences of gold under $\mathrm{CO}_{2} \mathrm{RR}$ conditions compared to cathodic corrosion during HER. Although strategies involving strongly alkaline electrolytes have been reported as a means of boosting the overall performance of $\mathrm{CO}_{2}$-to- $\mathrm{CO}$ electrocatalytic systems, ${ }^{36}$ the electrolyte conditions $(10 \mathrm{M} \mathrm{NaOH})$ are not representative of the most commonly reported conditions in the established literature (generally in the 0.1-0.5 $\mathrm{M} \mathrm{KHCO}_{3}$ range). ${ }^{37}$ It does, however, provide for an interface where $\mathrm{pH}$ changes due to changes in current can be neglected (whereas the interfacial $\mathrm{pH}$ of bicarbonate electrolytes is known to deviate significantly as a function of current density). ${ }^{38}$ Furthermore, it significantly enhances the rate at which a surface undergoes changes under cathodic potentials, which allows for shorter experiments.

It is important to note that cathodic corrosion in general is more prevalent at higher cation concentrations, although it has been shown to also occur at lower cation concentrations, albeit at a slower rate. ${ }^{11,33}$ As for gold specifically, there are currently no corrosion data for less concentrated electrolytes $(\leq 1 \mathrm{M})$, but the data available in the literature indicate that gold and platinum exhibit comparable behavior in the sense that corrosion is less pronounced at lower cation concentrations (but still takes place) and that similar types of sites are created irrespective of cation concentration, although the final state of the surface will likely still be different. ${ }^{11}$

In Figure $7 \mathrm{a}-\mathrm{d}$, cyclic voltammograms obtained after corrosion experiments in the absence (solid lines) and presence (dashed lines) of CO are depicted for the $\{111\}$, $\{100\}$, and $\{110\}$ basal planes and spherical single-crystalline surfaces. A close-up of the double-layer region for this figure, which encompasses all cyclic voltammograms discussed in this work, can be found in the Supporting Information (Figure S5). It is found that all surfaces exhibit the same trend when comparing cyclic voltammograms of crystals corroded in the absence of $\mathrm{CO}$ with cyclic voltammograms of crystals corroded in the presence of $\mathrm{CO}$, and therefore, only one facet will be discussed. As the CO effect is most pronounced for the $\{100\}$ facet (Figure $7 \mathrm{~b}$ ), that face will be discussed. When comparing the solid orange line ( $\mathrm{CV}$ after $2.5 \mathrm{~min}$ corrosion at $-1.2 \mathrm{~V}_{\mathrm{RHE}}$

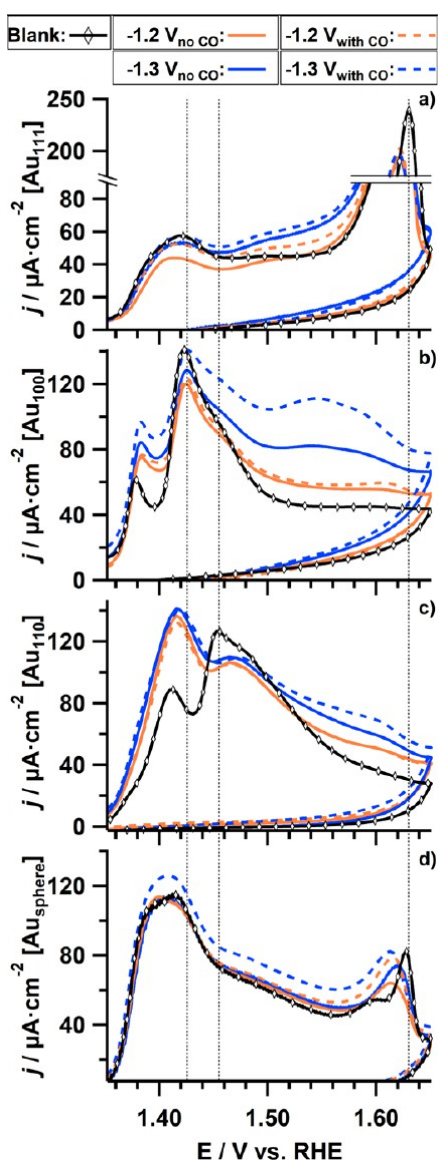

Figure 7. Cyclic voltammograms of gold single crystals in $0.05 \mathrm{M}$ $\mathrm{H}_{2} \mathrm{SO}_{4}$ after corroded in $10 \mathrm{M} \mathrm{NaOH}$ for $2.5 \mathrm{~min}$ in the absence (solid lines) and presence (dashed lines) of $\mathrm{CO}$ at $-1.2 \mathrm{~V}_{\mathrm{RHE}}$ (orange) and $-1.3 \mathrm{~V}_{\mathrm{RHE}}$ (blue) (85\% iR-corrected) for (a) $\mathrm{Au}(111),(\mathrm{b}) \mathrm{Au}(100),(\mathrm{c}) \mathrm{Au}(110)$, and (d) $\mathrm{Au}$ (spherical) electrodes, compared with their respective cyclic voltammograms prior to corrosion (black with a diamond).

without $\mathrm{CO}$ ) with the dashed orange line ( $\mathrm{CV}$ after corrosion with $\mathrm{CO}$ ), it can be seen that there is a slight excess in charge when $\mathrm{CO}$ was present during corrosion. The same is observed when comparing the solid blue curve ( $\mathrm{CV}$ after $2.5 \mathrm{~min}$ corrosion at $-1.3 \mathrm{~V}_{\mathrm{RHE}}$ without $\mathrm{CO}$ ) with the dashed blue line (corroded with CO present) albeit more pronounced. However, although the charge under the curves is found to increase (indicative of an increase in active sites), the overall shape remains mostly the same except for some small changes in the stepped 111 region. Hence, it is concluded that the presence of $\mathrm{CO}$ enhances the rate of corrosion, but the types of sites that are generated during the corrosion process are very similar, with perhaps a small increase in stepped 111 sites. This conclusion is generalizable to the three basal planes as they show the same trend as discussed for the $\{100\}$ face. Spherical crystal surfaces are also found to be similarly influenced by the presence of $\mathrm{CO}$, but from these measurements, it is not possible to ascertain whether this is applicable to all the facets that constitute the overall surface or whether all the changes are solely due to enhanced corrosion of the $\{111\},\{100\}$, and $\{110\}$ sites that are present.

A more quantitative proof for the effect of $\mathrm{CO}$ on cathodic corrosion is shown in Figure S6, wherein the roughness factors (RFs) for the crystals corroded under different conditions are depicted. Depending on the exact conditions and initial surface 


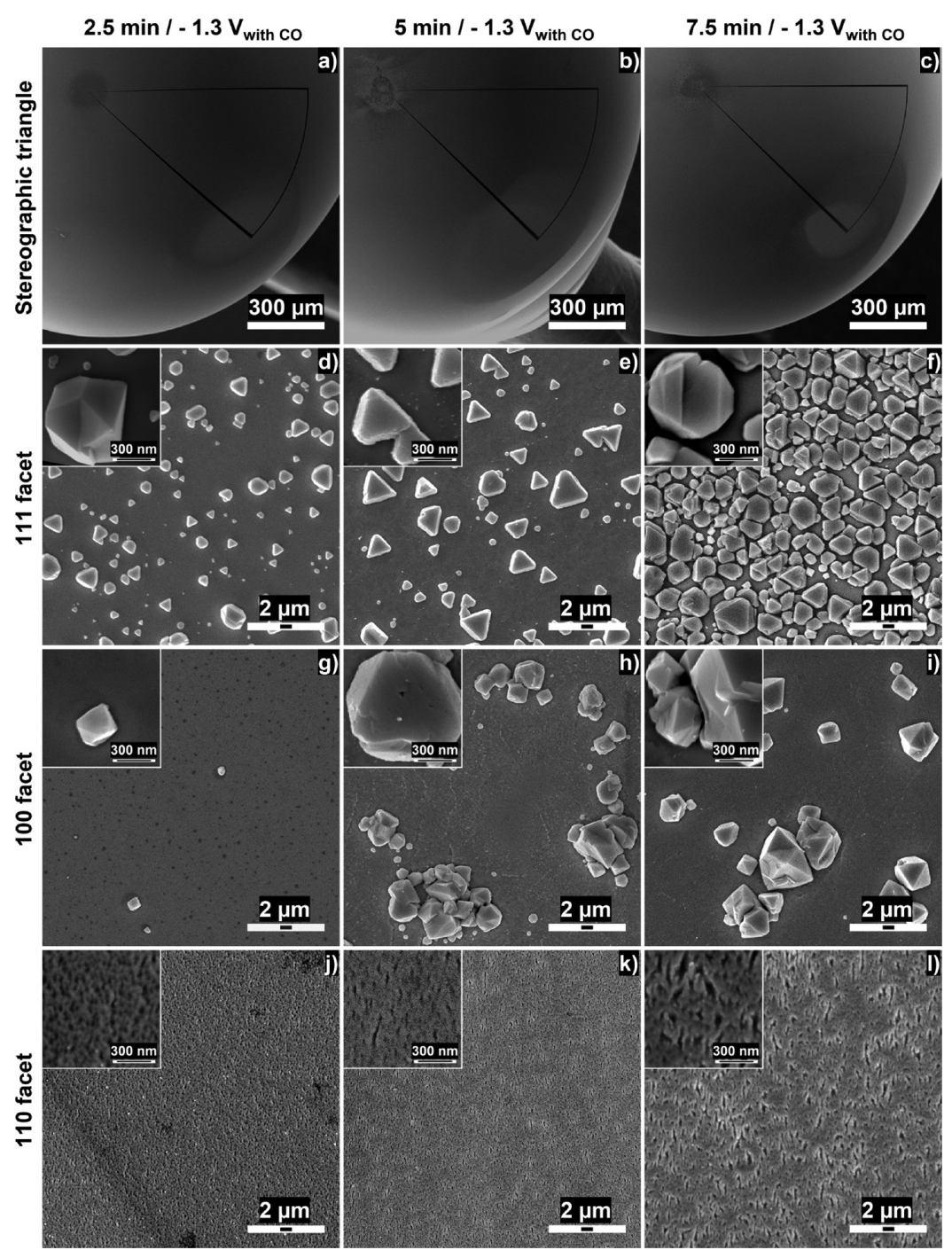

Figure 8. SEM images (macroscopic overview, the 111 facet, 100 facet, and 110 facet, going from the first row to the last row) of anisotropic surface corrosion in the form of pitting and nanocrystal formation as a function of time $(2.5-7.5 \mathrm{~min}$, going from the left column to the right column) at a corrosion potential of $-1.3 \mathrm{~V}_{\mathrm{RHE}}(85 \%$ iR-corrected) in $10 \mathrm{M} \mathrm{NaOH}$ with $\mathrm{CO}$ present.

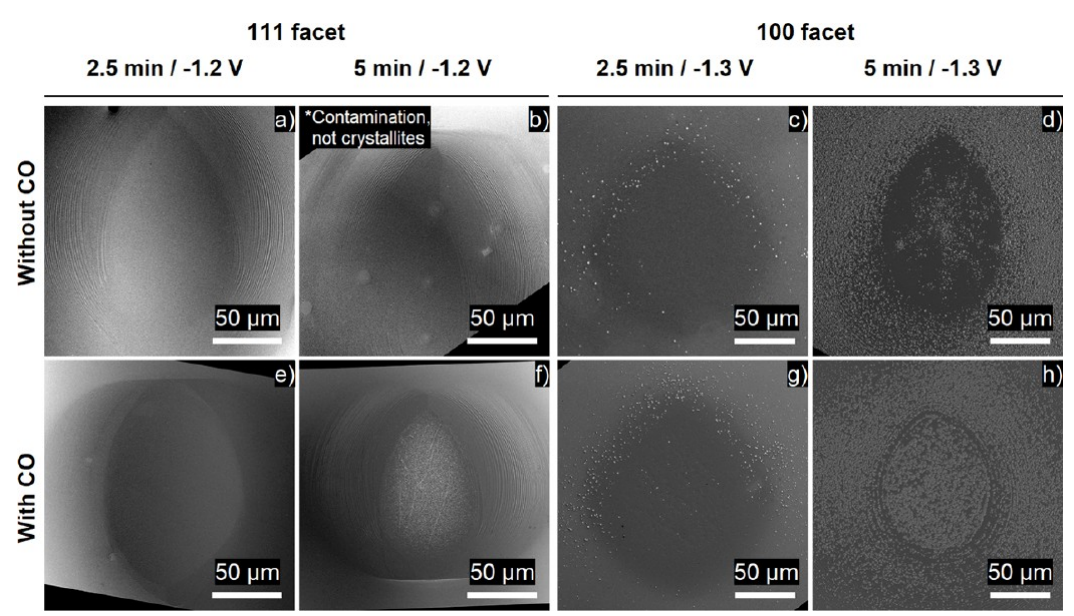

Figure 9. Corrosion-time-dependent nanocrystal growth during corrosion in the absence $(a-d)$ and presence $(e-h)$ of $\mathrm{CO}$ at a potential of -1.2 $\mathrm{V}_{\mathrm{RHE}}\left(85 \%\right.$ iR-corrected) for the 111 facet $(\mathrm{a}, \mathrm{b}$ and $\mathrm{e}, \mathrm{f})$ and $-1.3 \mathrm{~V}_{\mathrm{RHE}}(85 \%$ iR-corrected) for the 100 facet $(\mathrm{c}, \mathrm{d}$ and $\mathrm{g}, \mathrm{h})$.

structure, corrosion in the presence of carbon monoxide yields an increase of $2-20 \%$ in surface area post corrosion, compared to the surface area after corrosion in the absence of CO.
However, because the effect can be seen to be highly dependent on the initial surface structure and applied potential, we believe that it is more appropriate to describe 


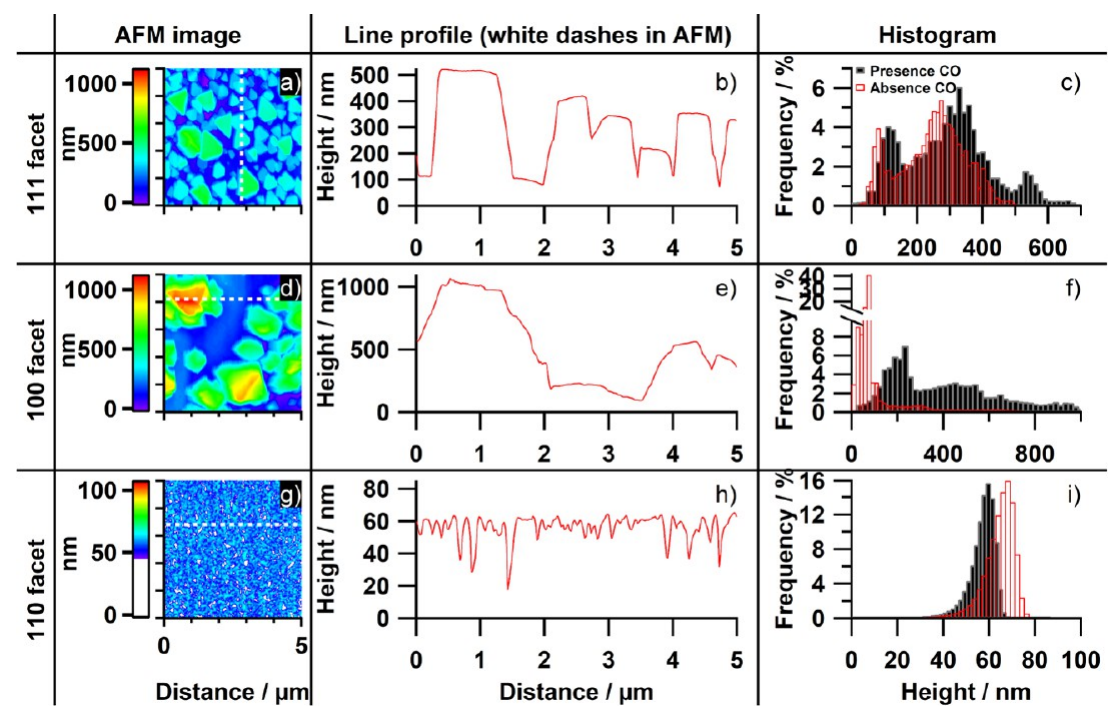

Figure 10. AFM images, line profiles, and histograms (columns 1, 2, and 3, respectively) for the basal planes $[\{111\}(\mathrm{a}-\mathrm{c}),\{100\}(\mathrm{d}-\mathrm{f})$, and $\{110\}$ $(\mathrm{g}-\mathrm{i})]$ after cathodic corrosion at $-1.3 \mathrm{~V}_{\mathrm{RHE}}(85 \%$ iR-corrected) for $5 \mathrm{~min}$ in $10 \mathrm{M} \mathrm{NaOH}$ with $\mathrm{CO}$ present.

the effect in qualitative terms rather than quantitative terms and hence refer to it as a "mild enhancement".

3.5.2. Morphological Characterization of the CO Effect on Cathodic Corrosion. 3.5.2.1. SEM Characterization of the CO Effect on Cathodic Corrosion. In Figure 8, an image is depicted, which is mostly identical to Figure 4, with the only change being that corrosion was conducted in the presence of $\mathrm{CO}$ as opposed to Ar. The same observations hold as made for surfaces corroded in the absence of CO. When comparing these two data sets, it is important to be aware of the fact that each SEM image only depicts a selected spot on the surface that we deemed to best portray the particular features of that face. Most notably, on this length scale, there is some variability in nanocrystal density. Hence, these particular sets of images (Figures 4 and 8) do not allow for a fair comparison of the average number of nanocrystals per unit area, for which the reader is, instead, referred to Figure 9.

Clear evidence for the slight enhancement of surface dissolution in the presence of $\mathrm{CO}$ is provided in the SEM images depicted in Figure 9. From images 9a,b, it can be seen that no nanocrystal growth is observed for $t_{\mathrm{p}} \leq 5 \mathrm{~min}$ at a corrosion potential of $-1.2 \mathrm{~V}_{\mathrm{RHE}}$ when $\mathrm{CO}$ is absent. However, nanocrystals are found to be present after $5 \mathrm{~min}$ when $\mathrm{CO}$ is present during corrosion under otherwise identical conditions. Although the gold ions that redeposit in the form of nanocrystals likely originate from elsewhere on the surface, the fact that crystallite growth is observed sooner does indicate enhanced dissolution in the presence of CO. Similar behavior is observed for nanocrystal growth on the $\{100\}$ facet (compare Figure 9c,d with $9 \mathrm{~g}, \mathrm{~h}$ ) for more negative applied corrosion potentials $\left(-1.3 \mathrm{~V}_{\mathrm{RHE}}\right)$. However, in both instances, this trend is only observed for crystallite formation near the observed onset of growth and does not apply to longer time frames and/or more corrosive potentials.

3.5.2.2. AFM Characterization of the CO Effect on Cathodic Corrosion. AFM images obtained upon corrosion in the presence of carbon monoxide are depicted in Figure 10. Observations pertaining to surface roughening and crystallite shapes are in line with those previously discussed for corrosion in the absence of $\mathrm{CO}$. However, the influence of $\mathrm{CO}$ on the growth of nanocrystals on the $\{111\}$ and $\{100\}$ faces can clearly be seen from the histograms. In both instances [Figure 10c for $\mathrm{Au}(111)$ and $10 \mathrm{f}$ for $\mathrm{Au}(100)$ ], the histograms show that in the absence of $\mathrm{CO}$, nanocrystals are less high. Thus, the presence of $\mathrm{CO}$ yields deposits with similar shapes but with an increased height. The face that does not exhibit crystallite growth $[\mathrm{Au}(110)]$ does not display a strong $\mathrm{CO}$ effect, exhibiting only small changes in the histogram on the order of ca. $10 \mathrm{~nm}$.

We attribute the slight enhancement of surface corrosion in the presence of $\mathrm{CO}$ to the interaction of carbon monoxide with the gold atoms at the surface. As $\mathrm{CO}$ is known to bind quite strongly to gold atoms in alkaline media, ${ }^{39,40}$ the surface mobility of these gold atoms is expected to increase (as binding an adsorbate decreases the binding strength a gold atom has with its neighboring gold atoms). ${ }^{41,42}$ Such effects can negatively influence catalyst stability under cathodic conditions in an industrial setting as many possible adsorbates will reasonably be present.

\section{DISCUSSION AND CONCLUSIONS}

In this work, cathodic corrosion has been studied on gold surfaces with well-defined surface structures so as to determine the types of surface sites that may be preferentially created when specific types of facets corrode. Specifically, CV was utilized to study changes in the hydroxide/oxygen adsorption region in $0.05 \mathrm{M} \mathrm{H}_{2} \mathrm{SO}_{4}$ after corrosion. Voltammetric changes of cut single crystals of the three basal planes, $\{111\},\{100\}$, and $\{110\}$, and those of (uncut) spherical single-crystalline surfaces were investigated and compared. The inclusion of spherical single-crystal electrodes enabled us to study further using SEM not only the macroscopic morphological changes of the three basal planes with corrosion potential $\left(E_{\mathrm{p}}\right)$ and corrosion time $\left(t_{\mathrm{p}}\right)$ but also the effect of the specific type and density of step defects present in a specific terrace. Additional information regarding the nano-/microscale surface morphology for the three basal planes was obtained from AFM imaging. Finally, all parameters were investigated for surfaces corroded in the absence and presence of carbon monoxide during corrosion. The inclusion of $\mathrm{CO}$ as a parameter serves as a controlled test environment that is meant to approximate, in part, the local environment of a gold surface if it were to be 
utilized in a $\mathrm{CO}_{2}$ electrolyzer. This parameter serves as an indication whether cathodic corrosion is influenced by the reaction that occurs at the interface [although the absence of $\mathrm{CO}_{2}$ (g) and bicarbonate/carbonate salts are artificially imposed (over)simplifications].

From CV, SEM, and AFM, it was found that cathodic corrosion is anisotropic, that is, dependent on the specific facet of the surface. CV data showed that corrosion of $\{100\}$ facets results in the preferential creation of stepped $\{111\}$ surface sites, and the same holds true for corrosion of the $\{110\}$ plane. Because of the fact that the $\{111\}$ face itself also undergoes corrosion (also resulting in a surface that voltammetrically resembles a stepped $\{111\}$ surface), it is concluded that under cathodic conditions, the surface changes in such a way that more stepped $\{111\}$ sites are created at the cost of other types of surface sites. The presence of $\mathrm{CO}$ during corrosion seems to accelerate corrosion slightly (as derived from CV charges), but the overall shape of the hydroxide/oxygen adsorption region remained very similar, except for possibly a slight increase in the fraction of stepped $\{111\}$. Therefore, the presence of carbon monoxide enhances the rate of corrosion only mildly and does not lead to a substantial shift in the types of sites that are created.

A further proof for anisotropic corrosion is obtained from both SEM and AFM by looking specifically at corrosion of the three basal planes. On two of these faces $(\{111\}$ and $\{100\})$, nanocrystal growth is observed after certain threshold potentials and corrosion times are exceeded, whereas crystallite formation is absent entirely for the $\{110\}$ facet. Additionally, nanocrystal shapes, spatial distribution, and the onset of formation are also facet-dependent. Specifically, nanocrystal shape-like triangular prisms that are distributed uniformly on the surface form on the $\{111\}$ face, whereas smaller individual octahedra and clusters of irregularly shaped crystallites form on the $\{100\}$ face. Interestingly, nanocrystal formation on the $\{100\}$ facet requires longer corrosion times and more corrosive potentials than that on $\{111\}$. Also, near the onset, crystallite formation on $\mathrm{Au}(100)$ is actually observed to start at the edges of this facet on a spherical single crystal, where $\{100\}$ facets with a low step density are located. This is in contrast with nanocrystal formation on the $\{111\}$ face, which only takes place on the step-defect-free facet. Supplementary differences between the crystallites that form on the two basal planes are obtained from AFM, which shows that the nanocrystals that grow on the $\{111\}$ face are terminated with a flat top that runs parallel to the base, much like a plateau, as opposed to the agglomerates that form on the $\{100\}$ face which are better described as irregular mountains. Furthermore, AFM has shown that nanocrystallites that form under corrosion in the presence of $\mathrm{CO}$ are higher than crystallites that form in the absence of CO. In a similar direction, SEM data show that the onset of crystallite growth is shifted to shorter corrosion times or yields higher nanocrystallite densities for the same corrosion times (limited to specifically time frames near the onset of crystallite formation), when $\mathrm{CO}$ is present during corrosion.

Contrary to the nanocrystal formation observed on $\mathrm{Au}(111)$ and $\mathrm{Au}(100)$, the $\{110\}$ face exhibits strong pitting and no crystallite growth under corrosive conditions. Furthermore, the formation of pits is observed to occur at less strongly corrosive applied potentials and shorter corrosion times from SEM than the conditions required for nanocrystal formation (and surface pitting) on the other two basal planes.
In conclusion, cathodic corrosion of gold can be divided into two corrosion modes: dissolution and pitting, and redeposition. As our experiments show nanocrystallite formation to be a function of corrosion time, we conclude that there must exist a concentration gradient that builds up over time, resulting into electrocrystallization at specific surface sites when a certain critical value is reached. Rather than gold ions dissolving and redepositing on the same face, SEM imaging shows that facets containing $\{110\}$ sites (both terraces and steps) and facets with increasing amounts of step-site defects exhibit more roughening upon corrosion than the $\{111\}$ and $\{100\}$ terraces with fewer to no step-site defects (which are the facets where nanocrystal growth occurs). Hence, we conclude that electrodissolution of more corrosive facets ( $\{110\}$ containing and terraces with step defects) results in the buildup of a concentration gradient of gold ions near the surface, which subsequently recrystallize on the faces that are less corrosive (defect-free $\{111\}$ and $\{100\}$ ). The fact that the presence of $\mathrm{CO}$ enhances corrosion rates leads to this concentration gradient building up faster and hence can explain a shift in the onset for crystallite formation. Presumably, the effect of $\mathrm{CO}$ is related to the chemisorption of $\mathrm{CO}$ on gold in alkaline media, ${ }^{39,40}$ thereby slightly weakening the $\mathrm{Au}-\mathrm{Au}$ bond and/or enhancing the gold atom surface diffusion. ${ }^{41,42}$

Anisotropic cathodic corrosion has also been observed for Pt electrodes in alkaline media. ${ }^{12,43}$ However, cathodic corrosion of platinum leads to substantially different surface transformations. For platinum, the $\{111\}$ facet is the most prone to cathodic corrosion, and $\{110\}$ the least, and leads to the preferential formation of $\{100\}$ surface sites, both steps and terraces. Only etch pits are observed on Pt, without evidence for redeposition, with a shape reflecting the atomic order of the surface. The observed structures on platinum suggest that the etching process is (surface-)diffusion-limited. The fact that gold exhibits very different behavior may suggest that the intermediate of cathodic corrosion (presumably a ternary metal hydride) has a different structure or stability for gold and that the role of mass transport in the generation of the surface roughness is different.

Finally, our experiments have shown that cathodic corrosion is a highly destructive phenomenon that severely impacts the structure of electrodes at negative potentials, specifically during electrocatalytic reduction reactions, such as $\mathrm{CO}_{2} \mathrm{RR}$, which run at a relatively high overpotential. This may undermine the stability of such electrodes in real electrolyzers as cathodically induced dissolution leads to consumption of the catalyst and furthermore, both dissolution and electrocrystallization phenomena result in preferential types of sites being produced, which may not necessarily be the most catalytically active sites for the reaction of interest as different facets generally exhibit different catalytic performances. ${ }^{44}$ On the other hand, leaving aside the stability aspect, cathodic corrosion may also lead to a surface site distribution that is actually beneficial for catalysis. ${ }^{45}$ We note that this study was conducted in a highly concentrated alkaline solution to accelerate cathodic corrosion and that surface changes are expected to be less severe if electrolytes with lower cation concentrations are employed.

\section{ASSOCIATED CONTENT}

\section{SI Supporting Information}

The Supporting Information is available free of charge at https://pubs.acs.org/doi/10.1021/acs.jpcc.0c08574. 
Onset of crystallite formation on the 111 facet and the onset of pitting for the 110 facet, as visible in SEM; proof for dissolution of the 111 and 100 facets, as visible in SEM; location of crystallite formation on the 100 facet near the observable onset, as visible in SEM; absence of nanocrystallite formation during long-term corrosion at $-1 \mathrm{~V}_{\mathrm{RHE}}$, as (in)visible in SEM; close-up of the double-layer region for cyclic voltammograms described in this work; and roughness factors of $\mathrm{Au}$ electrodes corroded under different conditions (PDF)

\section{AUTHOR INFORMATION}

\section{Corresponding Author}

Marc T. M. Koper - Leiden Institute of Chemistry, Leiden University, 2300 RA Leiden, The Netherlands; 1 orcid.org/ 0000-0001-6777-4594; Email: m.koper@lic.leidenuniv.nl

\section{Authors \\ Stefan J. Raaijman - Leiden Institute of Chemistry, Leiden University, 2300 RA Leiden, The Netherlands \\ Nakkiran Arulmozhi - Leiden Institute of Chemistry, Leiden University, 2300 RA Leiden, The Netherlands}

Complete contact information is available at:

https://pubs.acs.org/10.1021/acs.jpcc.0c08574

\section{Author Contributions}

${ }^{\dagger}$ S.J.R. and N.A. contributed equally to this work.

\section{Notes}

The authors declare no competing financial interest.

\section{ACKNOWLEDGMENTS}

This project is a part of the Solar-to-Products program financed by The Netherlands Organization for Scientific Research (NWO) and by Shell Global Solutions International B.V. This work also received partial support by Hitachi HighTech Corporation.

\section{REFERENCES}

(1) Warner, K.; Jones, G. The climate-independent need for renewable energy in the 21st century. Energies 2017, 10, 1197.

(2) Cave, E. R.; Montoya, J. H.; Kuhl, K. P.; Abram, D. N.; Hatsukade, T.; Shi, C.; Hahn, C.; Nørskov, J. K.; Jaramillo, T. F. Electrochemical $\mathrm{CO} 2$ reduction on Au surfaces: mechanistic aspects regarding the formation of major and minor products. Phys. Chem. Chem. Phys. 2017, 19, 15856-15863.

(3) Lu, Q.; Rosen, J.; Zhou, Y.; Hutchings, G. S.; Kimmel, Y. C.; Chen, J. G.; Jiao, F. A selective and efficient electrocatalyst for carbon dioxide reduction. Nat. Commun. 2014, 5, 3242.

(4) Azuma, M.; Hashimoto, K.; Hiramoto, M.; Watanabe, M.; Sakata, T. Electrochemical Reduction of Carbon Dioxide on Various Metal Electrodes in Low-Temperature Aqueous KHCO3 Media. J. Electrochem. Soc. 1990, 137, 1772-1778.

(5) Min, X.; Kanan, M. W. Pd-Catalyzed Electrohydrogenation of Carbon Dioxide to Formate: High Mass Activity at Low Overpotential and Identification of the Deactivation Pathway. J. Am. Chem. Soc. 2015, 137, 4701-4708.

(6) Dinh, C.-T.; Burdyny, T.; Kibria, M. G.; Seifitokaldani, A.; Gabardo, C. M.; García de Arquer, F. P.; Kiani, A.; Edwards, J. P.; De Luna, P.; Bushuyev, O. S.; et al. CO2 electroreduction to ethylene via hydroxide-mediated copper catalysis at an abrupt interface. Science 2018, 360, 783-787.

(7) Li, C. W.; Ciston, J.; Kanan, M. W. Electroreduction of carbon monoxide to liquid fuel on oxide-derived nanocrystalline copper. Nature 2014, 508, 504-507.
(8) Verma, S.; Kim, B.; Jhong, H.-R. M.; Ma, S.; Kenis, P. J. A. A Gross-Margin Model for Defining Technoeconomic Benchmarks in the Electroreduction of CO2. ChemSusChem 2016, 9, 1972-1979.

(9) Yanson, A. I.; Rodriguez, P.; Garcia-Araez, N.; Mom, R. V.; Tichelaar, F. D.; Koper, M. T. M. Cathodic Corrosion: A Quick, Clean, and Versatile Method for the Synthesis of Metallic Nanoparticles. Angew. Chem. Int. Ed. 2011, 50, 6346-6350.

(10) Arulmozhi, N.; Esau, D.; Lamsal, R. P.; Beauchemin, D.; Jerkiewicz, G. Structural Transformation of Monocrystalline Platinum Electrodes upon Electro-oxidation and Electro-dissolution. ACS Catal. 2018, 8, 6426-6439.

(11) Hersbach, T. J. P.; McCrum, I. T.; Anastasiadou, D.; Wever, R.; Calle-Vallejo, F.; Koper, M. T. M. Alkali Metal Cation Effects in Structuring $\mathrm{Pt}, \mathrm{Rh}$, and Au Surfaces through Cathodic Corrosion. ACS Appl. Mater. Interfaces 2018, 10, 39363-39379.

(12) Hersbach, T. J. P.; Yanson, A. I.; Koper, M. T. M. Anisotropic etching of platinum electrodes at the onset of cathodic corrosion. Nat. Commun. 2016, 7, 12653.

(13) Jouny, M.; Luc, W.; Jiao, F. General Techno-Economic Analysis of CO2 Electrolysis Systems. Ind. Eng. Chem. Res. 2018, 57, 21652177.

(14) Peng, X.; Karakalos, S. G.; Mustain, W. E. Preferentially Oriented Ag Nanocrystals with Extremely High Activity and Faradaic Efficiency for $\mathrm{CO} 2$ Electrochemical Reduction to CO. ACS Appl. Mater. Interfaces 2018, 10, 1734-1742.

(15) Štrbac, S.; Adžć, R. R.; Hamelin, A. Oxide formation on gold single crystal stepped surfaces. J. Electroanal. Chem. Interfacial Electrochem. 1988, 249, 291-310.

(16) Hamelin, A.; Martins, A. M. Cyclic voltammetry at gold singlecrystal surfaces. Part 2. Behaviour of high-index faces. J. Electroanal. Chem. 1996, 407, 13-21.

(17) Hamelin, A. Cyclic voltammetry at gold single-crystal surfaces. Part 1. Behaviour at low-index faces. J. Electroanal. Chem. 1996, 407, $1-11$.

(18) Herrero, E.; Buller, L. J.; Abruña, H. D. Underpotential Deposition at Single Crystal Surfaces of $\mathrm{Au}, \mathrm{Pt}, \mathrm{Ag}$ and Other Materials. Chem. Rev. 2001, 101, 1897-1930.

(19) Kibler, L. A. Preparation and characterization of noble metal single crystal electrode surfaces. Int. Soc. Electrochem. 2003, 1-55.

(20) Hersbach, T. J. P.; Mints, V. A.; Calle-Vallejo, F.; Yanson, A. I.; Koper, M. T. M. Anisotropic etching of rhodium and gold as the onset of nanoparticle formation by cathodic corrosion. Faraday Discuss. 2016, 193, 207-222.

(21) Medina-Ramos, J.; Zhang, W.; Yoon, K.; Bai, P.; Chemburkar, A.; Tang, W.; Atifi, A.; Lee, S. S.; Fister, T. T.; Ingram, B. J.; et al. Cathodic Corrosion at the Bismuth-Ionic Liquid Electrolyte Interface under Conditions for $\mathrm{CO} 2$ Reduction. Chem. Mater. 2018, 30, 2362-2373.

(22) Kaischew, R.; Mutaftschiew, B. Uber die elektrolytische keimbildung des quecksilbers. Electrochim. Acta 1965, 10, 643-650.

(23) Clavilier, J.; Faure, R.; Guinet, G.; Durand, R. Preparation of monocrystalline Pt microelectrodes and electrochemical study of the plane surfaces cut in the direction of the $\{111\}$ and $\{110\}$ planes. $J$. Electroanal. Chem. Interfacial Electrochem. 1980, 107, 205-209.

(24) Arulmozhi, N.; Jerkiewicz, G. Design and Development of Instrumentations for the Preparation of Platinum Single Crystals for Electrochemistry and Electrocatalysis Research. Part 1: SemiAutomated Crystal Growth. Electrocatalysis 2016, 7, 507-518.

(25) Arulmozhi, N.; Esau, D.; van Drunen, J.; Jerkiewicz, G. Design and Development of Instrumentations for the Preparation of Platinum Single Crystals for Electrochemistry and Electrocatalysis Research Part 3: Final Treatment, Electrochemical Measurements, and Recommended Laboratory Practices. Electrocatalysis 2018, 9, 113123.

(26) Trasatti, S.; Petrii, O. A. Real surface area measurements in electrochemistry. J. Electroanal. Chem. 1992, 327, 353-376.

(27) Einstein, T. L., 5 - Equilibrium Shape of Crystals. In Handbook of Crystal Growth, 2nd ed.; Nishinaga, T., Ed.; Elsevier: Boston, 2015; pp 215-264. 
(28) Cherevko, S.; Zeradjanin, A. R.; Keeley, G. P.; Mayrhofer, K. J. J. A Comparative Study on Gold and Platinum Dissolution in Acidic and Alkaline Media. J. Electrochem. Soc. 2014, 161, H822-H830.

(29) Schalenbach, M.; Kasian, O.; Ledendecker, M.; Speck, F. D.; Mingers, A. M.; Mayrhofer, K. J. J.; Cherevko, S. The Electrochemical Dissolution of Noble Metals in Alkaline Media. Electrocatalysis 2018, 9, $153-161$

(30) Feng, J.; Chen, D.; Sediq, A. S.; Romeijn, S.; Tichelaar, F. D.; Jiskoot, W.; Yang, J.; Koper, M. T. M. Cathodic Corrosion of a Bulk Wire to Nonaggregated Functional Nanocrystals and Nanoalloys. ACS Appl. Mater. Interfaces 2018, 10, 9532-9540.

(31) Parker, S. F. Spectroscopy and bonding in ternary metal hydride complexes-Potential hydrogen storage media. Coord. Chem. Rev. 2010, 254, 215-234.

(32) Abrahams, S. C.; Ginsberg, A. P.; Knox, K. Transition MetalHydrogen Compounds. II. The Crystal and Molecular Structure of Potassium Rhenium Hydride. Inorg. Chem. 1964, 3, 558-567.

(33) Yanson, A. I.; Antonov, P. V.; Rodriguez, P.; Koper, M. T. M. Influence of the electrolyte concentration on the size and shape of platinum nanoparticles synthesized by cathodic corrosion. Electrochim. Acta 2013, 112, 913-918.

(34) Personick, M. L.; Mirkin, C. A. Making Sense of the Mayhem behind Shape Control in the Synthesis of Gold Nanoparticles. J. Am. Chem. Soc. 2013, 135, 18238-18247.

(35) Seifitokaldani, A.; Gabardo, C. M.; Burdyny, T.; Dinh, C.-T.; Edwards, J. P.; Kibria, M. G.; Bushuyev, O. S.; Kelley, S. O.; Sinton, D.; Sargent, E. H. Hydronium-Induced Switching between $\mathrm{CO} 2$ Electroreduction Pathways. J. Am. Chem. Soc. 2018, 140, 3833-3837.

(36) Gabardo, C. M.; Seifitokaldani, A.; Edwards, J. P.; Dinh, C.-T.; Burdyny, T.; Kibria, M. G.; O’Brien, C. P.; Sargent, E. H.; Sinton, D. Combined high alkalinity and pressurization enable efficient $\mathrm{CO} 2$ electroreduction to CO. Energy Environ. Sci. 2018, 11, 2531-2539.

(37) Nitopi, S.; Bertheussen, E.; Scott, S. B.; Liu, X.; Engstfeld, A. K.; Horch, S.; Seger, B.; Stephens, I. E. L.; Chan, K.; Hahn, C.; et al. Progress and Perspectives of Electrochemical CO2 Reduction on Copper in Aqueous Electrolyte. Chem. Rev. 2019, 119, 7610-7672.

(38) Gupta, N.; Gattrell, M.; MacDougall, B. Calculation for the cathode surface concentrations in the electrochemical reduction of $\mathrm{CO} 2$ in KHCO3 solutions. J. Appl. Electrochem. 2006, 36, 161-172.

(39) Kita, H.; Nakajima, H.; Hayashi, K. Electrochemical oxidation of $\mathrm{CO}$ on $\mathrm{Au}$ in alkaline solution. J. Electroanal. Chem. Interfacial Electrochem. 1985, 190, 141-156.

(40) Rodriguez, P.; Garcia-Araez, N.; Koverga, A.; Frank, S.; Koper, M. T. M. CO Electroxidation on Gold in Alkaline Media: A Combined Electrochemical, Spectroscopic, and DFT Study. Langmuir 2010, 26, 12425-12432.

(41) Besenbacher, F.; Lægsgaard, E.; Stensgaard, I. Fast-scanning STM studies. Mater. Today 2005, 8, 26-30.

(42) Wang, J.; McEntee, M.; Tang, W.; Neurock, M.; Baddorf, A. P.; Maksymovych, P.; Yates, J. T. Formation, Migration, and Reactivity of $\mathrm{Au}-\mathrm{CO}$ Complexes on Gold Surfaces. J. Am. Chem. Soc. 2016, 138, $1518-1526$.

(43) Arulmozhi, N.; Hersbach, T. J. P.; Koper, M. T. M. Nanoscale Morphological Evolution of Monocrystalline Pt Surfaces during Cathodic Corrosion. Proc. Natl. Acad. Sci. U.S.A. 2020, 202017086.

(44) Mezzavilla, S.; Horch, S.; Stephens, I. E. L.; Seger, B.; Chorkendorff, I. Structure Sensitivity in the Electrocatalytic Reduction of CO2 with Gold Catalysts. Angew. Chem. 2019, 131, 3814-3818.

(45) Duca, M.; Rodriguez, P.; Yanson, A. I.; Koper, M. T. M. Selective Electrocatalysis on Platinum Nanoparticles with Preferential (100) Orientation Prepared by Cathodic Corrosion. Top. Catal. 2014, $57,255-264$. 